\title{
Experimental study of Taylor's hypothesis in a turbulent soap film
}

\author{
Andrew Belmonte*, Brian Martin, and Walter I. Goldburg \\ Department of Physics and Astronomy, University of Pittsburgh, Pittsburgh, PA 15260, U. S. A.
}

\begin{abstract}
An experimental study of Taylor's hypothesis in a quasi-two-dimensional turbulent soap film is presented. A two probe laser Doppler velocimeter enables a non-intrusive simultaneous measurement of the velocity at spatially separated points. The breakdown of Taylor's hypothesis is quantified using the cross correlation between two points displaced in both space and time; correlation is better than $90 \%$ for scales less than the integral scale. A quantitative study of the decorrelation beyond the integral scale is presented, including an analysis of the failure of Taylor's hypothesis using techniques from predictability studies of turbulent flows. Our results are compared with similar studies of 3D turbulence.
\end{abstract}

PACS Number(s): 47.27.Gs, 68.15.+e, 03.40.Gc

REVISED VERSION

(April 5, 2018)

\section{INTRODUCTION}

In a 1938 paper on the statistics of turbulence, G. I. Taylor presented an assumption from which he could infer the spatial structure of a turbulent velocity field from a single point measurement of its temporal fluctuation [1]. This assumption, known as Taylor's hypothesis or the frozen turbulence assumption, relies on the existence of a large mean flow which translates the fluctuations past the stationary probe in a time short compared to the evolution time of the turbulence. The experimental measurements treated by Taylor were made on the turbulence generated behind a stationary grid in a wind tunnel, and his hypothesis has become a standard technique employed in similar experiments which inform our current views on turbulence (see for example Refs. [2], [3], and [4]). The importance of this hypothesis stems from the fact that most turbulence theories are framed in terms of the spatial structure of the velocity field [2,5].

In practical terms, the limits of Taylor's hypothesis are determined by how large the mean velocity must be relative to the fluctuations. Recently Yakhot [6] has pointed out that the corrections to Taylor's hypothesis could well be of the same order as corrections to the standard model of turbulence, Kolmogorov's 1941 theory [7,8]. There is at present, however, no firm theoretical derivation of the hypothesis, and thus no fashion to evaluate its reliability, calculate higher order corrections, or predict in what way it will break down.

*Present address: Department of Mathematics, Pennsylvania State University, University Park, PA 16802, USA. 
A few theoretical discussions do exist [9 [1], and a promising direction has recently been taken by Hayot \& Jayaprakash for the Burgers equation [12. The treatment by Lumley in particular offers corrections to statistical measures of spatial gradients for non-negligible velocity fluctuations [10]. Still a general theoretical framework is lacking.

Experimentally there have been many studies of Taylor's hypothesis, all of which have treated three dimensional (3D) turbulence; we give a brief overview in Section II. Our experiments, however, are performed on the quasi-two dimensional flow of a soap film. Twodimensional (2D) fluid flows occur in many physical situations, mostly due to the effects of rotation or stratification in the atmosphere and ocean $[13]$. Turbulence in $2 \mathrm{D}$ is different from 3D in several ways, largely due to the absence of vortex stretching in 2D [14]. This means that the squared vorticity (called enstrophy) becomes a nearly-conserved quantity in 2D, like the energy, and thus two cascades are expected: a direct cascade of enstrophy to smaller scales, and an inverse cascade of energy to larger scales [15]; for decaying 2D turbulence the inverse cascade is apparently absent [14,16]. Although Taylor's hypothesis is also an important assumption in the study of 2D turbulence, to our knowledge it has never been tested, nor is it clear that the hypothesis should do relatively better or worse than in 3D.

It may be helpful at this point to discuss the essential differences between $2 \mathrm{D}$ and $3 \mathrm{D}$ turbulence in order to better relate the present measurements to prior three dimensional tests of the Taylor hypothesis. In three dimensions the vorticity vector $\omega(x, y, z, t)$ can point in any direction, but in 2D it is restricted to be perpendicular to the $x, y$ plane of the flow. This fact alone assures that in inviscid flows, the enstrophy $\left.\Omega=\frac{1}{2}<\omega^{2}\right\rangle$ is a constant of the motion, in addition to kinetic energy conservation $K=\frac{1}{2}<v^{2}>$ (the angular brackets designate an appropriate average). From the Navier-Stokes equation it follows that in 2D, vorticity cannot be amplified (or attenuated except by viscous damping) by a velocity field gradient. The existence of vortex stretching in 3D is intimately related to the energy cascade from large scales to small, with the process controlled by the rate $\epsilon$ at which kinetic energy is injected at large scales.

In $2 \mathrm{D}$, one expects that energy injected at intermediate scale, $r_{i n j}$ will be transferred to larger scales [14, 17] and dissipated at the boundaries of the system. This inverse cascade process is not expected to be local in k-space. For scales $r<r_{i n j}$, the small-scale velocity fluctuations $<|\delta v(r)|>$, which carry little of the energy, are expected to cascade down to the dissipative scale under the control of the enstrophy injection rate $\beta \equiv \partial \Omega / \partial t$ (the energy injection rate $\epsilon$ plays the corresponding role in the 2D inverse cascade).

It seems reasonable that the absence of vortex stretching in $2 \mathrm{D}$ will increase the likelihood of a velocity fluctuation being transported intact to a distant downstream point, thereby enhancing the validity of Taylor's hypothesis over 3D turbulence. Taylor's hypothesis can also fail when a local fluctuation is transported laterally into the flow path between adjacent points separated by the distance $\Delta x=U_{0} \Delta t$. For purely geometrical reasons, this would seem to be a less likely occurence in $2 \mathrm{D}$ than in $3 \mathrm{D}$, so that the frozen turbulence relation $\delta v(\Delta x)=\delta v\left(U_{0} t\right)$ should presumably hold out to larger values of $\Delta x$. It is harder for us to assess the impact of $2 \mathrm{D}$ nonlocality on the validity of the Taylor hypothesis in $2 \mathrm{D}$ as compared to 3D.

Our experiment uses a laser Doppler velocimeter (LDV) with two probes to nonintrusively examine Taylor's hypothesis in a turbulent soap film. We differentiate between two different 
aspects of the hypothesis. By Taylor's hypothesis of coherence we mean the assertion that the velocity field is unchanged as it is advected downstream: that $v(x, t)$ is identical to $v\left(x+\Delta x, t+\tau_{0}\right)$, where $\tau_{0}=\Delta x / U_{0}$, with $U_{0}$ being the mean velocity and $\Delta x$ the distance between the two points [1]. For a clear illustration of this, glance ahead to Figure 9. This coherence hypothesis is unquestionably an approximation and must fail as $\Delta x$ becomes large; here we quantify this failure, and relate it to the predictability problem for turbulent flows. We measure the breakdown of the coherence hypothesis via the correlation between two points in the flow, displaced in both position and time [18 23]. We find nevertheless that the expectation value of the lowest six moments of the longitudinal velocity difference is unaffected by the loss of coherence. Thus time correlation statistics of velocity fluctuations appear to be the same as spatial ones; we will refer to this as Taylor's statistical hypothesis, which is implied by the coherence hypothesis, but does not actually require it.

\section{PREVIOUS STUDIES OF TAYLOR'S HYPOTHESIS}

Taylor's hypothesis has been exposed to many experimental tests in three dimensional turbulence, and we do not intend to give an exhaustive survey here. These experimental studies can be divided into two broad categories, concerned with either correlations over finite distances [18 23], or local spatial derivatives used in turbulent dissipation estimates 24 27. It has long been appreciated that the validity of Taylor's frozen turbulence assumption requires the smallness of the turbulent intensity $I_{t}$, defined as the ratio of rms velocity fluctuations to the mean flow speed $U_{0}$. Additionally, the mean shear rate and the viscous damping must be small in the range of spatial scales $\Delta x$ being probed. In this section we give a sampling of the sort of work which has been done in 3D; a more detailed discussion of studies with which we directly compare our results is given in Section V. The reader is referred to introductory reviews in two recent articles [26.27], which are somewhat complementary to what is given here.

To test the application of Taylor's hypothesis to velocity correlations over finite distances, one approach is to compare measurements made at a single observation point with measurements made at points displaced downstream. The first such tests were made in a wind tunnel by Favre, Gaviglio, \& Dumas [18,19]. The measurements were performed within the turbulent boundary layer of a plate at various $I_{t}$ up to $15 \%$. They found that Taylor's statistical hypothesis is valid for measurements of the velocity correlation function $R(\Delta x, \tau)=\left\langle v_{1}\left(x_{1}, t\right) v_{2}\left(x_{1}+\Delta x, t-\tau\right)\right\rangle$ made not too close to the plate. Fisher \& Davies [20] made careful measurements of the velocity correlation function $R(\Delta x, \tau)$ in a jet. They found that the relation $\tau=\Delta x / U_{0}$ was not well satisfied in the mixing region, where $I_{t}$ is typically $\sim 20 \%$. These authors observed, as have many others, that the functional form of $R$ changes with increasing $\Delta x$, and that this function is not very sharply peaked, as it would be if Taylor's coherence hypothesis were satisfied. Comte-Bellot \& Corrsin [22] measured $R(\Delta x, \tau)$ for grid-generated turbulence in a wind tunnel, where both $I_{t}$ and the mean shear rates are rather small. Though downstream decay causes the maximum value of $R$ vs. $\tau$ to decrease with increasing $\Delta x$, the correlation functions could still be collapsed onto the same functional form.

One of the fundamental effects of turbulence is its enhancement of dissipation, and measurements of this require knowledge of spatial derivatives. Using Taylor's hypothesis allows 
the time deriative of a single point measurement to be related to spatial derivatives, the simplest relation being $\partial \phi / \partial x=U_{0} \partial \phi / \partial t$, where $\phi$ could be a passive scalar concentration, temperature, a velocity component, or a product of velocity components. Kailasneth, Sreenivasan, \& Saylor [25] studied a variety of turbulent systems and tested Taylor's hypothesis using a fluorescent dye in a jet, the heated wake of a cylinder, and the atmospheric boundary layer. They were interested in the probability density function of these scalars, and found that Taylor's statistical hypothesis worked well for conditional probability densities of the scalar fluctuations. Mi \& Antonia [26] used a heated jet of air, with a turbulent intensity of about $26 \%$, to verify different theoretical relations between spatial and temporal derivatives of temperature, corrected for finite turbulent intensities. Dahm \& Southerland [27] compared 2D spatial and spatio-temporal gradient fields of fluorescent dye in a water jet using a fast photodiode array. They found that Taylor's coherence hypothesis was only approximately verified for these fields. Piomelli, Balint, \& Wallace [24 studied Taylor's hypothesis for various velocity derivatives and compared hot wire measurements, large eddy simulations, and direct numerical simulations of the Navier-Stokes equation for wall-bounded flows. Taylor's hypothesis was found to be in accord with the calculations and measurements made sufficiently far from the wall, where the mean shear is not excessive. In our experiments we have not treated the application of Taylor's hypothesis to gradients.

All hot wire measurements are sufficiently intrusive that one must compensate or otherwise adjust for the perturbations produced by the wake of the upstream probe on the velocity measured at the downstream probe. Cenedese et al. [23] avoided this problem by making velocity measurements with a Laser Doppler system. Only for small $\Delta x$ did their correlation measurements satisfy Taylor's hypothesis very well, though admittedly $I_{t}$ was rather high (13\%). We discuss their results in more detail in Section V.

In the present experiment, Laser Doppler velocimetry is also used to measure $R(\Delta x, \tau)$, so that there is no perturbation on the downstream probe. In contrast to the studies discussed above, however, we examine Taylor's hypothesis in a quasi-2D system, a flowing soap film; we have also studied the effects on the higher order moments of velocity differences. Before presenting our results, we discuss in some detail our experimental system.

\section{EXPERIMENTAL SETUP}

The use of soap films as convenient systems for the experimental study of 2D hydrodynamics began with the pioneering work of Couder and coworkers [28 30] and Gharib and Derango [31]. Our measurements are performed using a flowing soap film apparatus developed at the University of Pittsburgh by Kellay, Wu, \& Goldburg [32], and Rutgers, Wu, \& Goldburg [33].34]; we are using the latest version of this system, built by Rutgers. In our setup, a thin soap film several $\mu \mathrm{m}$ thick is allowed to fall between two taut plastic wires from an upper reservoir into a lower one, see Figure ]. The channel width is $W=6.2 \mathrm{~cm}$ over a distance of $120 \mathrm{~cm}$, where the measurements are performed. Quasi-two-dimensional turbulence is generated behind a comb (tooth diameter $1 \mathrm{~mm}$ and spacing $M=3.8 \mathrm{~mm}$ ) which perforates the film at a fixed height. The typical transit time between comb and lower reservoir is the order of a second.

The turbulence generated in such a soap film decays downstream from the grid, exhibiting

many aspects which agree with theories of $2 \mathrm{D}$ turbulence [29 32,35. 36], though there are also 
some differences. It therefore seems worthwhile to evaluate the experimental situation to date. Because the lateral dimensions of soap films are many orders of magnitude larger than their thickness, there would seem to be no doubt that the vorticity can indeed be regarded as a scalar quantity, so that vortex stretching is absent [5]. This would be a key requirement for the occurence two-dimensional turbulence in soap films. In addition, to be able to compare with theoretical and numerical results concerning incompressible turbulence, the two-dimensional compressibility of the film should be zero. This condition is clearly not fully met, since fluctuations in film thickness are visible to the eye through optical interference of light reflected from the front and back faces of the film [30,31,36]. There are, however, recent experiments in which the two dimensional divergence $D_{2}=\nabla_{2} \dot{\mathbf{v}}(x, y)$ was measured by particle imaging velocimetery [44. In a set-up very similar to that used here, $D_{2}$ was measured to be 10-15\% of the rms vorticity near the comb. Note also that because the velocity of peristaltic waves in a soap film is orders of magnitude larger than the turbulent velocity fluctuations [30], it is expected that the film may be regarded as incompressible from the point of view of this study.

Another mitigating factor to the two-dimensionality of soap film flow is the friction between the film and the surrounding air. In a set of experiments in which the film was placed in a partial vacuum where the air pressure $3 \%$ of the atmospheric value, it was found that the energy spectrum $E(k)$ decayed for a decade in $k$ as $E(k) \propto k^{-\zeta}$, with $\zeta=3.3 \pm 0.3$. This exponent had the same value in both the partial vacuum and at atmospheric pressure. The main effect of the reduced pressure was to magnify the magnitude of $E(k)$ near the comb [35]. These measurements also showed that the total kinetic energy initially decayed downstream, but ultimately leveled off at a sufficient distance below the comb, which is expected theoretically at very high Reynolds numbers [16]. If the levelling off distance is called $x^{*}$ and the corresponding time $t^{*}=x^{*} / U_{0}$, then all measurements reported in this paper were made at values of $t<t^{*}$, i.e. at distances from the comb too close for the levelling off to have occurred.

In most (but not all) measurements of decaying turbulence in a soap film, only the enstrophy cascade is observed, i.e. the inverse energy cascade $\left(E(k) \sim k^{-5 / 3}\right)$ is not. Recently an experiment was performed in which turbulence was forced by an array of teeth parallel to the direction of flow [36]. There one finds evidence of both the inverse cascades $\left(k^{-5 / 3}\right)$ and the inverse cascade spectrum, where $k^{-3}$.

We use a commercial LDV system [37] to measure the film velocity fluctuations [38, 39]. The soap solution (water and $2 \%$ commercial detergent by volume) is seeded with $1 \mu \mathrm{m}$ polystyrene spheres at a volume fraction of about $10^{-4}$, and the data rate ranges from 1 to $8 \mathrm{kHz}$. At a distance $x=8 \mathrm{~cm}$ below the comb, the mean and RMS velocities were typically $U_{0}=180 \mathrm{~cm} / \mathrm{s}$ and $v_{R M S} \equiv\left\langle v^{\prime 2}\right\rangle^{1 / 2}=24 \mathrm{~cm} / \mathrm{s}$ in the longitudinal (streamwise) direction, where $v^{\prime} \equiv v-U_{0}$. The turbulent intensity in these experiments was $I_{t} \simeq 0.14$. This is the quantity which is assumed in Taylor's hypothesis to be small [1,9], and we have explicitly chosen for our study a value of $I_{t}$ which is not very small. The Reynolds number for the channel is $R e_{W} \equiv U_{0} W / \nu \simeq 11,000$, and for the comb $R e_{M} \equiv U_{0} M / \nu \simeq 700$. The viscosity of a flowing soap film is not a well established quantity; here we use $\nu=0.1 \mathrm{~cm}^{2} / \mathrm{s}$ as measured in a 2D Couette viscometer by Martin \& Wu [40]. Deviations from twodimensionality caused by air friction [34] appear not to affect the turbulence for the scales of interest here [35]. 
In order to test Taylor's hypothesis, two LDV heads are used at spatially separated points. Figure 1 shows the arrangement, with the downward (flow) direction defined as the $x$ direction. One head (labeled "LDV 1") is kept fixed at $x_{1}=8 \mathrm{~cm}$ below the comb, while a second head ("LDV 2") is placed at various points ranging from $x_{2}=4$ to $30 \mathrm{~cm}$ below the comb. The two probes are arranged to be directly in line with each other, so that the lower probe measures the same part of the flow as the upper one, with a delay given by the transit time between them.

Because the LDV only measures velocity when there is a scatterer in its measuring volume, the two probes do not in general measure velocity simultaneously. Therefore some binning or "simultaneity window" $\Delta t$ is needed to perform statistical comparisons: two measurements are treated as simultaneous if they occur within $\Delta t$ of one another. Here we use binning windows from $\Delta t=25 \mu \mathrm{s}$ to $200 \mu \mathrm{s}$, corresponding to frequencies $1 / \Delta t$ from 5 to $40 \mathrm{kHz}$, which are higher than the largest observed frequency in the velocity power spectrum. All measurements reported here are insensitive to small changes in $\Delta t$.

One of the difficulties in examining the validity of Taylor's hypothesis in decaying turbulence is that the "small parameter" $I_{t}$ is not constant, but decreases downstream as the turbulence decays. In Fig. Ba we plot our turbulent intensity as a function of distance from the comb. Thus, although we measure the correlation of velocity fluctuations relative to $x_{1}=8 \mathrm{~cm}$, where $I_{t} \simeq 0.14$, the actual turbulent intensity affecting the velocity field downstream is always less than 0.14 . This would seem to significantly complicate the matter.

How does the turbulence decay in our system? A standard result from 3D decaying grid turbulence is that the inverse square of the turbulent intensity depends on the distance from

the grid as: $I_{t}^{-2}=A(x / M-B)^{\beta}$, where the dimensionless constants are typically found to be $A \sim 130-150$ and $B \sim 3-20$ for $\beta=1$ [41], or $A \sim 20$ and $B \sim 3.5$ for $\beta=1.25$ [22]; note that $B$ is the effective position of the origin for this scaling in units of $M$. Assuming that $\beta=1.25$ means that $I_{t}^{-1.6}$ should be a linear function of $x / M$; however we find that by taking $I_{t}^{-1.1}$ we get the best linear plot vs. $x / M$, as shown in Fig. $8 \mathrm{~b}$. The line corresponds to

$$
\frac{1}{I_{t}^{2}}=0.2\left(\frac{x}{M}\right)^{1.8},
$$

with $B=0$ for our fit, which means that the virtual origin is located at the position of the grid itself. Note that we do not measure far enough downstream 35] to see any evidence of the kinetic energy saturation as the turbulence decays downstream $\left(I_{t} \sim\right.$ constant $)$.

\section{RESULTS}

\section{A. Testing Taylor's Coherence Hypothesis}

Figure 9 shows the velocity fluctuations measured by two probes with $\Delta x=0.5 \mathrm{~cm}$. For this small separation, Taylor's hypothesis is clearly a good estimate: the two velocity traces are nearly identical except for a small shift in time, which should correspond to the transit time across the spatial separation $\Delta x$. To test whether the velocity trace is translated spatially without evolving dynamically, we measure the cross-correlation $C_{12}\left(\tau, \Delta x, x_{1}\right)$ between the two probes: 


$$
C_{12}\left(\tau, \Delta x, x_{1}\right) \equiv \frac{\left\langle v_{1}\left(x_{1}, t\right) v_{2}\left(x_{1}+\Delta x, t-\tau\right)\right\rangle}{v_{1 R M S} \times v_{2 R M S}} .
$$

Here $v_{1}\left(x_{1}, t\right)$ and $v_{2}\left(x_{2}, t\right)$ are the two velocities measured by the probes, $v_{i R M S}$ are the RMS velocity fluctuations, $\Delta x \equiv x_{2}-x_{1}$, and the brackets \langle\rangle denote a time average. Note that $C_{12}$ is a function not only of delay time $\tau$ and separation $\Delta x$, but of the absolute location of the first probe $x_{1}$. This last dependence comes from the fact that the turbulence is decaying. In this study we fix $x_{1}=8 \mathrm{~cm}$, and ignore this dependence.

Figure 10 shows $C_{12}(\tau, \Delta x)$ vs. $\tau$ for several different separations $\Delta x$. As expected, there is a well-defined maximum correlation

$$
C_{12}^{M A X}(\Delta x) \equiv C_{12}\left(\tau_{M A X}, \Delta x\right)
$$

at a particular value of the delay time $\tau_{M A X}(\Delta x)$. Taylor's coherence hypothesis requires that $C_{12}^{M A X}(\Delta x)$ be close to 1 , and $\tau_{M A X}(\Delta x)$ be equal to the transit time $\Delta x / U_{0}$. Figure 11 shows $\tau_{M A X}$ as a function of $\Delta x / U_{0}$, in agreement with the line drawn for $\tau_{M A X}=\Delta x / U_{0}$ [42]. As predicted by Taylor's hypothesis, the slope of this line is unity. The small deviations are due to errors in our measurement of $\Delta x$.

In Figure 12 we plot the maximum correlation $C_{12}^{M A X}(\Delta x)$. As one would expect, the correlation decreases as $\Delta x$ increases, though we have not found any simple functional form to fit to this decrease, nor is there to our knowledge any predicted form. The loss of correlation is due to the dynamic evolution of the velocity fluctuations and sets a limit to Taylor's hypothesis, which we quantify by defining an "evolution length" $\delta_{e}$ as the separation for which the correlation drops to $50 \%$. For our experiment we find $\delta_{e} \simeq 7 \mathrm{~cm}$, corresponding to an evolution time $\tau_{e}=\delta_{e} / U_{0} \simeq 40 \mathrm{~ms}$. This length is much larger than the relevant lengths of the turbulent velocity field, as we show next.

\section{B. Testing Taylor's Statistical Hypothesis}

The statistical study of turbulence is framed in terms of velocity correlation functions, structure functions, and energy spectra; here we focus on the structure function. The longitudinal velocity difference between two points separated by a distance $r$ is written as

$$
\delta v(r, t) \equiv\left(\mathbf{v}\left(x_{1}+r, t\right)-\mathbf{v}\left(x_{1}, t\right)\right) \cdot \hat{\mathbf{r}},
$$

where the unit vector $\hat{\mathbf{r}}$ is in the downward direction of the flow. The $n$th order structure function is defined as:

$$
S_{n}(r) \equiv\left\langle(\delta v(r, t))^{n}\right\rangle .
$$

In Figure 13 we show the structure functions $S_{2}(r), S_{4}(r)$, and $S_{6}(r)$, calculated from single point velocity measurements using Taylor's hypothesis: $r=U_{0} \tau$ (solid circles). We also plot direct spatial measurements of these structure functions made using two probes (open squares). This is a direct confirmation of Taylor's statistical hypothesis, which is one of the central results of our study. Note that $S_{2}(r)$ shows a scaling region of about a decade where $S_{2}(r) \propto r^{1.6}$, in good agreement with other experiments on turbulent soap films [43, 44]. We also find approximately that $S_{4}(r) \propto r^{2.9}$ and $S_{6}(r) \propto r^{4.0}$, as shown in the figure. The third moment $S_{3}(r)$ has been treated in detail elsewhere 43 . 
Some comment must be made on the observed values of the exponents, which are so different from $S_{n}(r) \propto r^{n}$, the theoretical expectation for the enstrophy cascade in 2D turbulence [16, 15]. It is well known that in 3D turbulence the scaling law exponents of the $n$th order structure functions deviate from their expected value of $n / 3$ as $n$ gets large [45]. This systematic difference is attributed to the intermittency of the fluctuations [5]. However, the third order structure function must scale as $S_{3}(r) \sim r$ even with intermittency, as can be derived directly from the Navier-Stokes equations [5]. An equivalent derivation for 2D turbulence would not be relevant to the third moment in the enstrophy cascade range discussed here. In fact $S_{3}$ is observed to be approximately zero in this range (and is positive for large $r$ ) [43]. This observation suggests to us that the scaling exponents of the enstrophy range are more sensitive to intermittency than in 3D turbulence. Evidence of intermittency, indicated by non-Gaussian velocity fluctuations, has been reported previously for our experiment [43].

For $r>1 \mathrm{~cm}$, the $S_{n}(r)$ saturate to constant values, which for $S_{2}(r)$ is equal to $2 v_{R M S}^{2}$. This occurs roughly at the integral or outer scale [2], which characterizes the largest scales on which the velocity is correlated. The integral scale $\ell_{0}$ is defined as

$$
\ell_{0} \equiv \int_{0}^{\infty} b(r) d r / v_{R M S}^{2}
$$

where $b(r)$ is the velocity correlation function:

$$
b(r) \equiv\left\langle v^{\prime}(x, t) v^{\prime}(x+r, t)\right\rangle=v_{R M S}^{2}-\frac{1}{2} S_{2}(r) .
$$

At $x_{1}=8 \mathrm{~cm}$ we find $\ell_{0}=0.6 \mathrm{~cm}$, which is much less than the evolution length $\delta_{e}=7 \mathrm{~cm}$. Thus for the turbulence in our soap film, Taylor's hypothesis is justified: the two signals are correlated better than $90 \%$ for scales $r<\ell_{0}$ (see Figs. 12 and 13).

\section{Detailed Study of the Velocity Decorrelation}

There are in general two reasons for the breakdown of Taylor's hypothesis: the entrance of new structures into the line of travel, introducing new fluctuations into the signal, and the evolution of the velocity field itself. In Figure 14 we show an overlay of the velocity measured at $x_{1}=8.0 \mathrm{~cm}$ vs. $t$, and the velocity measured at $x_{2}=10.0 \mathrm{~cm}$ vs. $t-\tau_{M A X}$ $(\Delta x=2 \mathrm{~cm})$. For a perfect correlation $\left(C_{12}^{M A X}=1\right)$ the two curves would fall on top of each other. The arrows indicate fluctuations which have either appeared or disappeared during the transit time between the two probes. In effect this means that information is being generated, and this "new information" is partially responsible for the velocity decorrelation (Fig. 5).

To explore the details of this process, we measure the coherence spectrum $C s(f)$ of the fluctuations at $x_{1}$ and $x_{2}$ [46]. If Taylor's hypothesis of coherence were justified, then $v_{1} \equiv v\left(x_{1}, t\right)$ would be identical to $v_{2} \equiv v\left(x_{2}, t-\tau_{M A X}\right)$. If $\hat{v}_{1}(f)$ is the complex Fourier transform of $v_{1}$, then the standard power spectrum is $P s_{1}(f)=\left\langle\hat{v}_{1}(f) \hat{v}_{1}^{*}(f)\right\rangle$, where $\hat{v}^{*}$ is the complex conjugate of $\hat{v}$. The coherence spectrum is

$$
C s(f) \equiv \frac{1}{2} \frac{\left\langle\hat{v}_{1}(f) \hat{v}_{2}^{*}(f)+\hat{v}_{1}^{*}(f) \hat{v}_{2}(f)\right\rangle}{\sqrt{P s_{1}(f) \times P s_{2}(f)}},
$$


normalized so that $C s=1.0$ for frequencies where the two time series are coherent. A measurement of $C s(f)$ with increasing probe separation shows which modes in the turbulent spectrum persist longer and which evolve faster.

Consider first the velocity power spectrum at a single point, shown as the thin line in Figure 15. This spectrum, according to the standard picture of $2 \mathrm{D}$ decaying turbulence [15,16], should have a power law dependence $P s(f) \sim f^{-\alpha}$ in the enstrophy cascade range, with $\alpha=3$. In an earlier soap film experiment [35], this exponent was found to be measurably larger than 3 ; here in we find $\alpha=3.6 \pm 0.2$. We compare this to the coherence spectrum, which we expect to be nearly equal to 1.0 for small separations. The coherence spectrum for $\Delta x=0.2 \mathrm{~cm}$ is also shown in Figure 15 (thick line). We see that $C s(f)$ is indeed close to unity over most of the frequency range in which the power spectrum appears. However, the coherence drops below $C s \sim 0.9$ at $f \sim 300 \mathrm{~Hz}$, around the middle of the range where $P s(f) \sim f^{-\alpha}$, and for $f \sim 850 \mathrm{~Hz}$, where the power spectrum is reaching the noise floor in our measurement, $C s \sim 0.2$. Thus already at $\Delta x=0.2 \mathrm{~cm}$ it appears that the high frequency components are the most rapidly evolving. In contrast, for the $2 \mathrm{D}$ enstrophy cascade it is expected that the 'eddy turnover time' is independent of size [14]; the observed falloff in $C s(f)$ may indicate viscous dissipation effects.

The coherence spectra at five increasing separations $\Delta x$ are shown in Figure 16, as a linear-log plot. In each case we see decorrelation at higher frequencies (smaller scales), while the low frequency part remains at a constant value which decreases as $\Delta x$ increases. This constant correlation is approximately equal to $C_{12}^{M A X}(\Delta x)$, which means that the overall coherence of the velocity field is determined mainly by the low frequency components. The high frequency decorrelation also moves to lower frequencies as $\Delta x$ increases. To see whether the whole shape of the $C s(f)$ follows the decay of $C_{12}^{M A X}(\Delta x)$, we normalize the coherence spectra as $C s(f) / C_{12}^{M A X}$ in Figure 17. The curves do not lie entirely on top of each other, indicating that the cutoff at high frequencies follows a different evolution than $C_{12}^{M A X}$. The cutoff shape is well described as $C s(f) \sim \log (1 / f)$, shown as the straight lines drawn through the data. This advancing cutoff is reminescent of the loss of predictability in the spectra of atmospheric turbulence simulations 14,47].

At larger separations $(\Delta x>12 \mathrm{~cm})$, we find that the spectral position of this cutoff no longer moves to lower frequencies as $\Delta x$ increases. In Figure 18 we superpose the coherence spectra for $\Delta x$ from 12 to $22 \mathrm{~cm}$, normalized by $C_{12}^{M A X}(\Delta x)$. The curves lie reasonably on top of each other, which means that the entire coherence spectrum follows the overall decrease of $C_{12}^{M A X}$. By taking the intersection of the logarithmic fit with the line $C s(f)=C_{12}^{M A X}(\Delta x)$ in Figs. 17 and 18, we use the frequency $f_{d}$ of the intersection to characterize the position of the spectral cutoff [14]. We plot this frequency in Figure 19]. Up to $\Delta x \simeq 12 \mathrm{~cm}$, the advance of $f_{d}$ to lower frequencies is consistent with the scaling $f_{d} \sim \Delta x^{-1 / 2}$, which is slower than that seen for wavenumber cutoff in 2D numerical simulations of atmospheric predictability [14,47]. For $\Delta x>12 \mathrm{~cm}, f_{d}$ reaches a constant value of about $30 \mathrm{~Hz}$, corresponding to a length of $6 \mathrm{~cm}$, which is the size of our system (the channel width $W=6 \mathrm{~cm}$ ).

\section{Turbulent Predictability and Taylor's Hypothesis}

The failure of Taylor's hypothesis in our experiment is closely related to the question of

predictability in 2D turbulence [47 49]. The general study of predictability in turbulence 
(see for example [14,50,51]) is of particular importance to the weather prediction problem [52. Here we briefly show how our analysis parallels this general framework. Note that here we are comparing a developing turbulent velocity with its initial state, whereas studies of predictability treat the diverging evolution of two nearly identical initial states. Nonetheless there are several similarities between the two.

Following Métais \& Lesieur, we first define the time series of the velocity difference, or error time series [47], which for our experiment is written

$$
\Delta v(\Delta x, t) \equiv v_{1}\left(x_{1}, t\right)-v_{2}\left(x_{1}+\Delta x, t-\tau_{M A X}\right) .
$$

For a perfectly correlated signal this time series would be identically zero. We define the difference energy $\Delta E(\Delta x)=\left\langle(\Delta v(\Delta x, t))^{2}\right\rangle$, which is the second moment of $\Delta v$ and thus a kinetic energy associated with the difference series; it is analogous to the error energy in predictability studies [47,54]. In Figure 20 we plot a dimensionless $\Delta E$, namely

$$
\rho(\Delta x) \equiv \frac{\Delta E(\Delta x)}{v_{1 R M S}^{2}+v_{2 R M S}^{2}},
$$

as a function of the decay time $\Delta x / U_{0}$. The function $\rho$ is defined to increase from 0 to 1 as $\Delta x$ increases, and acts as a sort of distance function between the two velocities. By comparing Eqs. 1 and 5, one sees that $\rho$ and $C_{12}$ are simply related.

The inset to Fig. 20 shows an enlargement of $\rho\left(\Delta x / U_{0}\right)$ near $\Delta x / U_{0}=0$. There is no clear linear portion in the plot which would correspond to an exponential error increase. In the study of turbulent predictability an exponential error growth is used to define a sort of Lyapunov exponent [53], with the error energy serving as a metric for evaluating the distance between co-evolving turbulent states. The data shown in Fig. 20 are in fact better described by a power law $\rho \sim\left(\Delta x / U_{0}\right)^{1 / 2}$, as shown in Fig. 21. This apparent square root dependence should be interpreted only as a power law dependence: the actual value of the exponent depends on the choice of metric function, Eq. 5. Since an exponential growth of the error energy depends on the linearization of an underlying equation for $\rho$, Fig. 21 may indicate the presence of higher order terms, similar to the quadratic saturation term used by Lorenz to fit error growth in an iterated map [55].

The predictability time $T_{p}$ is a standard measure of the time beyond which one can no longer project the state of a turbulent system [47,51]. The exact definition of such a time is somewhat arbitrary, though it is usually much larger than the large scale eddy turnover time for the turbulent flow [47]. We can characterize the long time growth of $\rho(\tau)$ by quantifying how long the velocity $v_{2}$ remains similar to $v_{1}$. The predictability time used by Métais \& Lesieur was defined by $\rho\left(T_{p}\right)=0.5$ [47]; we define an evolution time $T_{e}$ such that $\rho\left(T_{e}\right)=0.5$, and find that $T_{e} \simeq 25 \mathrm{~ms}$. This is of the same order as our decorrelation time $\tau_{e} \simeq 40 \mathrm{~ms}$ given by $C_{12}^{M A X}$ (Fig. 12), a result which is not surprising given that the two functions are related. The analogy between the loss of predictability and the failure of Taylor's hypothesis is in fact rooted in a common cause: the loss of velocity coherence due to turbulence. Whether any implications can be drawn from this connection remains to be seen.

\section{DISCUSSION}




\section{A. Comparison with 3D Measurements}

We have measured the breakdown of Taylor's hypothesis for decaying turbulence in a flowing soap film and shown that the hypothesis is a valid assumption for statistical measurements of the turbulence (the structure functions). How do our measurements compare to similar experimental studies of 3D decaying turbulence? Of the six studies which to our knowledge provide information comparable to Fig. 12 [18 23 we will examine three in detail [21 23]. Two of these studies used hot wire anemometry [21,22], and thus additional techniques were required to compensate for the wake of the upstream probe. Champagne et al. [21] used a 'grid' made of 12 parallel channels (spacing $M^{\prime}=2.54 \mathrm{~cm}$ ) in a wind tunnel with a mean speed of $12 \mathrm{~m} / \mathrm{s}$, and $R e_{M^{\prime}}=21,000$. Cross-correlation measurements started at $x_{1}=259 \mathrm{~cm}$, where $I_{t} \simeq 0.018$ and $\ell_{0} \simeq 4.2 \mathrm{~cm}$. Comte-Bellot \& Corrsin [22] made measurements behind a standard grid $(M=5.08 \mathrm{~cm})$ in a wind tunnel with $R e_{M}=$ 34,000. The two hot-wire cross correlation measurements were made starting at $x_{1}=210$ $\mathrm{cm}$, where $I_{t} \simeq 0.022$ and $\ell_{0} \simeq 1.1 \mathrm{~cm}$. Cenedese et al. [23] used a nonintrusive laser Doppler anemometer similar to our LDV (see [39]), but did not use a standard grid; the turbulence was produced by a combination of a honeycomb and the channel walls. Their measurements were made in a water channel (height $h=2 \mathrm{~cm}$ ) starting at $x_{1}=14 \mathrm{~cm}$, where $I_{t} \simeq 0.13$ and $\ell_{0} \simeq 1.0 \mathrm{~cm}$. Their Reynolds numbers were also significantly lower: $R e_{h}=4,800$.

Are there any differences between Taylor's hypothesis in our approximately twodimensional soap film and in these 3D experiments? We address this question by plotting $C_{12}^{M A X}(\Delta x)$ from these three studies along with our measurements in Figure 22. The independent variable in this plot is $\Delta x$ in units of the integral scale $\ell_{0}$. One might expect the decorrelation to occur more slowly in the soap film due to the absense of vortex stretching. However, as the turbulent intensity in our experiment is high $\left(I_{t}=0.14\right)$ compared to the two wind tunnel experiments $\left(I_{t} \sim 0.02\right)$, our data should be directly compared only to that of Cenedese et al. $\left(I_{t}=0.13\right)$. In this case we see that indeed the correlation in our soap film extends to much larger values of $\Delta x / \ell_{0}$ than in their $3 \mathrm{D}$ experiment. Note that $C_{12}^{M A X}$ from the wind tunnel experiments also extends to much larger values of $\Delta x / \ell_{0}$ than the data of Cenedese et al, probably due to the fact that their turbulent intensities are much lower. More definitive conclusions would come from a single experiment (in 2D or 3D) which measures $C_{12}^{M A X}(\Delta x)$ for several different $I_{t}$.

\section{B. Detailed Shape of the Cross Correlation $C_{12}(\tau)$}

As of yet there is no rigorous underpinning to Taylor's hypothesis which would allow for the calculation of higher order corrections to velocity correlations, though an intriguing suggestion was implemented in [56]. To provide detailed information for some future theory, we focus on the shape of the cross-correlation function $C_{12}(\tau, \Delta x)$ around $\tau_{M A X}$. This shape is by definition (Eq. 国) the average convolution of a velocity fluctuation taken with itself

a time $\tau_{M A X}$ later. In Figure 23 we show as an example $C_{12}(\tau)$ for $\Delta x=4 \mathrm{~cm}$, along with a Gaussian distribution centered on $\tau_{M A X}$. We find that the shape is always nearly Gaussian, with a slight skewness around $\tau_{M A X}$ consistently towards the positive. The width of the Gaussian does not broaden as $\Delta x$ increases, though the maximum does decrease as shown in Figure 24. Thus the development of the cross-correlation cannot be treated as a 
diffusion-like process, for which the width would increase as the maximum decreases. The small positive skewness is also not strongly dependent on $\Delta x$.

\section{CONCLUSION}

In this paper we focused on the breakdown of Taylor's coherence hypothesis in a turbulent soap film, a quasi-2D experimental system. We have shown that for the lower order moments the statistical hypothesis works well, even when the actual cross correlation between the

two probes is low. As the relevant length scale of this decorrelation is much larger than the integral scale of the turbulence $\left(\delta_{e}>>\ell_{0}\right)$, this phenomenon is outside the region usually considered by most studies: it is the turbulence beyond the scaling range. Yet this evolution contains untapped information, as we have indicated. The failure of Taylor's hypothesis may thus shed light on deeper problems in turbulence.

\section{ACKNOWLEDGEMENTS}

We would like to thank M. Rivera and X. L. Wu for beneficial and insightful discussions, and H. Kellay, M. A. Rutgers, R. Cressman, and the referees for helpful comments on the manuscript. This work was supported by NASA and the National Science Foundation. 


\section{REFERENCES}

[1] G. I. Taylor, "The spectrum of turbulence", Proc. Roy. Soc. London A 164, 476 (1938).

[2] A. S. Monin and A. M. Yaglom, Statistical Fluid Mechanics, (MIT Press, Cambridge, 1975), Vol. 2.

[3] A. Arneodo et al., "Structure functions in turbulence, in various flow configurations, at Reynolds number between 30 and 5000, using extended self-similarity", Europhys. Lett. 34, 411 (1996).

[4] K. R. Sreenivasan and R. A. Antonia, "The phenomenology of small-scale turbulence", Ann. Rev. Fluid Mech. 29, 435 (1997).

[5] U. Frisch, Turbulence, (Cambridge University Press, Cambridge, 1995).

[6] V. Yakhot, "On the relation between two-point and two-time correlation fluctuations in strong turbulence", preprint (1996); "Experiments on strong turbulence: do we really measure what we say we measure?", in Flow at Ultra-High Reynolds and Rayleigh Numbers: A Status Report, R. J. Donnelly and K. R. Sreenivasan, eds. (Springer, New York, 1998).

[7] A. N. Kolmogorov, "The local structure of turbulence in an incompressible fluid for very large Reynolds numbers", Dokl. Akad. Nauk. SSSR 30, 301 (1941), reprinted in Proc. Roy. Soc. London A 434, (1991).

[8] A. N. Kolmogorov, "Energy dissipation in locally isotropic turbulence", Dokl. Akad. Nauk. SSSR 32, 16 (1941), reprinted in Proc. Roy. Soc. London A 434, (1991).

[9] C. C. Lin, "On Taylor's hypothesis and the acceleration terms in the Navier-Stokes equations", Quart. Appl. Math. 10, 295 (1953).

[10] J. L. Lumley, "Interpretation of time spectra measured in high-intensity shear flows", Phys. Fluids 8, 1056 (1965).

[11] H. Tennekes, "Eulerian and Lagrangian time microscales in isotropic turbulence", $J$. Fluid Mech. 67, 561 (1975).

[12] F. Hayot and C. Jayaprakash, "Dynamical structure factors in models of turbulence", Phys. Rev. E 57, 4867 (1998).

[13] R. Salmon, Lectures in Geophysical Fluid Dynamics, (Oxford, New York, 1998).

[14] M. Lesieur, Turbulence in Fluids, (Kluwer Academic Publishers, Dordrecht, 1990), 2nd edition.

[15] R. Kraichnan, "Inertial ranges in two-dimensional turbulence", Phys. Fluids 10, 1417 (1967).

[16] G. K. Batchelor, "Computation of the energy spectrum in homogeneous two-dimensional turbulence", Phys. Fluids Suppl. II, 233 (1969).

[17] R. H. Kraichnan and D. Montgomery, Rep. Prog. Phys. 43, 547 (1980).

[18] A. J. Favre, J. J. Gaviglio, and R. Dumas, "Space-time correlations and spectra in a turbulent boundary layer", J. Fluid Mech. 2, 313 (1957).

[19] A. J. Favre, J. J. Gaviglio, and R. Dumas, "Further space-time correlations of velocity in a turbulent boundary layer", J. Fluid Mech. 3, 344 (1958).

[20] M. J. Fisher and P. O. A. L. Davies, "Correlation measurements in a non-frozen pattern of turbulence", J. Fluid Mech. 18, 97 (1964).

[21] F. H. Champagne, V. G. Harris, and S. Corrsin, "Experiments on nearly homogeneous turbulent shear flow", J. Fluid Mech. 41, 81 (1970). 
[22] G. Comte-Bellot and S. Corrsin, "Simple Eulerian time correlation of full- and narrowband velocity signals in grid-generated, 'isotropic' turbulence", J. Fluid Mech. 48, 273 (1971).

[23] A. Cenedese, G. P. Romano, and F. Di Felice, "Experimental testing of Taylor's hypothesis by L. D. A. in highly turbulent flow", Exp. in Fluids 11, 351 (1991).

[24] U. Piomelli, J.-L. Balint, and J. M. Wallace, "On the validity of Taylor's hypothesis for wall-bounded flows", Phys. Fluids A 1, 609 (1989).

[25] P. Kailasnath, K. R. Sreenivasan, and J. R. Saylor, "Conditional scalar dissipation rates in turbulent wakes, jets, and boundary layers", Phys. Fluids A 5, 3207 (1993).

[26] J. Mi and R. A. Antonia, "Corrections to Taylor's hypothesis in a turbulent circular jet", Phys. Fluids 6, 1548 (1994).

[27] W. J. A. Dahm and K. B. Southerland, "Experimental assessment of Taylor's hypothesis and its applicability to dissipation estimates in turbulent flows", Phys. Fluids 9, 2101 (1997).

[28] Y. Couder, "The observation of a shear flow instability in a rotating system with a soap membrane", J. Physique Lett. 42, 429 (1981).

[29] Y. Couder, "Two-dimensional grid turbulence in a thin liquid film", J. Physique Lett. 45, 353 (1984).

[30] Y. Couder, J.-M. Chomaz, and M. Rabaud, "On the hydrodynamics of soap films", Physica D 37, 384 (1989).

[31] M. Gharib and P. Derango, "A liquid film (soap film) tunnel to study two-dimensional laminar and turbulent shear flows", Physica D 37, 406 (1989).

[32] H. Kellay, X. L. Wu, and W. I. Goldburg, "Experiments with turbulent soap films", Phys. Rev. Lett. 74, 3975 (1995).

[33] W. I. Goldburg, M. A. Rutgers, and X. L. Wu, "Experiments on turbulence in soap films", Physica A 239, 340 (1997).

[34] M. A. Rutgers, X. L. Wu, R. Bagavatula, A. A. Peterson, and W. I. Goldburg, "Twodimensional velocity profiles and laminar boundary layers in flowing soap films", Phys. Fluids 8, 2847 (1997).

[35] B. Martin, X. L. Wu, W. I. Goldburg, and M. A. Rutgers, "Spectra of decaying turbulence in a soap film", Phys. Rev. Lett. 80, 3964 (1998).

[36] M. A. Rutgers, "Forced 2D turbulence: experimental evidence of simultaneous inverse energy and forward enstrophy cascades", Phys. Rev. Lett. 81, 2244 (1998).

[37] We are using two 9253-120 fiberoptic LDV probes, a Colorlink 9230, and an IFA 655 correlator from TSI Inc, P. O. Box 64204, St. Paul, MN 55164 USA.

[38] F. Durst, A. Melling, and J. H. Whitelaw, Principles and Practice of Laser-Doppler Anemometry, (Academic Press, New York, 1981), 2nd edition.

[39] P. Buchhave, W. K. George, and J. Lumley, "The measurement of turbulence with the laser-Doppler anemometer", Ann. Rev. Fluid Mech. 11, 443 (1979).

[40] B. Martin and X. L. Wu, "Shear flow in a two-dimensional Couette cell: A technique for measuring the viscosity of free-standing liquid films", Rev. Sci. Instr. 66, 5603 (1995).

[41] G. K. Batchelor and A. A. Townsend, "Decay of isotropic turbulence in the initial period", Proc. Roy. Soc. London A 193, 539 (1948).

[42] For these calculations we recalculate $U_{0}$ for each $\Delta x$ as the average of $\left\langle v_{1}\left(x_{1}, t\right)\right\rangle$ and $\left\langle v_{2}\left(x_{2}, t\right)\right\rangle$. 
[43] A. Belmonte, W. I. Goldburg, H. Kellay, M. A. Rutgers, B. Martin, and X. L. Wu, "Velocity fluctuations in a turbulent soap film: The third moment in two dimensions", Phys. Fluids 11, 1196 (1999).

[44] M. Rivera, P. Vorobieff, and R. E. Ecke, "Turbulence in flowing soap films: Velocity, vorticity, and thickness fields", Phys. Rev. Lett. 81, 1417 (1998).

[45] F. Anselmet, Y. Gagne, E. Hopfinger, and R. Antonia, J. Fluid Mech. 140, 63 (1984).

[46] G. M. Jenkins and D. G. Watts, Spectral Analysis and its Applications, (Holden-Day, San Francisco, 1968), Chapter 8.

[47] O. Métais and M. Lesieur, "Statistical predictability of decaying turbulence", J. Atmos. Sci. 43, 857 (1986).

[48] C. E. Leith, "Atmospheric predictability and two-dimensional turbulence", J. Atmos. Sci. 28, 145 (1971).

[49] S. Kida, M. Yamada, and K. Ohkitani, "Error growth in a decaying two-dimensional turbulence", J. Phys. Soc. Japan 59, 90 (1990).

[50] C. E. Leith and R. H. Kraichnan, "Predictability of turbulent flows", J. Atmos. Sci. 29, 1041 (1972).

[51] E. Aurell, G. Boffetta, A. Crisanti, G. Paladin, and A. Vulpiani, "Growth of noninfinitesimal perturbuations in turbulence", Phys. Rev. Lett. 77, 1262 (1996).

[52] Turbulence and Predictability in Geophysical Fluid Dynamics and Climate Dynamics, M. Ghil, R. Benzi, and G. Parisi, eds. (North-Holland, Amsterdam, 1985).

[53] J.-P. Eckmann and D. Ruelle, "Ergodic theory of chaos and strange attractors", Rev. Mod. Phys. 57, 617 (1985).

[54] G. Boffetta, A. Celani, A. Crisanti, and A. Vulpiani, "Predictability in two-dimensional decaying turbulence", Phys. Fluids 9, 724 (1997).

[55] E. N. Lorenz, "The growth of errors in prediction", in Ref. [52].

[56] J.-F. Pinton and R. Labbé, "Correction to the Taylor hypothesis in swirling flows", J. de Physique II (France) 4, 1461 (1994). 


\section{FIGURES}

FIG. 1. A diagram of the experimental setup: A) upper soap reservoir; B) hooks which hold plastic wires between which soap film flows; C) comb behind which turbulence is generated in the film; D) lower soap reservoir. The two LDV probes which measure the velocity in the film are labeled 1 and 2. Downstream distances are labeled as referred to in the text.

FIG. 2. The decay of the turbulent intensity $I_{t}$ behind the comb in our soap film: a) $I_{t}$ vs. downstream distance $x ; \mathrm{b}$ ) the same data plotted as $I_{t}^{-1.1}$ vs. $x / M$. The straight line is a linear fit (see text).

FIG. 3. Simultaneous traces of the velocity vs. time at $x_{1}=8.0 \mathrm{~cm}$ and $x_{2}=8.5 \mathrm{~cm}$ behind the comb.

FIG. 4. The cross correlation $C_{12}(\tau, \Delta x)$ vs. delay time $\tau$ for several different values of $\Delta x$ (as labeled).

FIG. 5. The delay time $\tau_{M A X}$ for the maximum of the cross correlations in Fig. 10 vs. the transit time $\Delta x / U_{0}$. The solid line corresponds to $\tau_{M A X}=\Delta x / U_{0}$.

FIG. 6. The maximum value of the cross correlations in Fig. 10, $C_{12}^{M A X}$ vs. the downstream separation $\Delta x$. The arrow shows the evolution length $\delta_{e} \simeq 7 \mathrm{~cm}$ (see text).

FIG. 7. A diagram of the experimental setup: A) upper soap reservoir; B) hooks which hold plastic wires between which soap film flows; C) comb behind which turbulence is generated in the film; D) lower soap reservoir. The two LDV probes which measure the velocity in the film are labeled 1 and 2. Downstream distances are labeled as referred to in the text.

FIG. 8. The decay of the turbulent intensity $I_{t}$ behind the comb in our soap film: a) $I_{t}$ vs. down-

stream distance $x ; \mathrm{b}$ ) the same data plotted as $I_{t}^{-1.1}$ vs. $x / M$. The straight line is a linear fit (see text). 
FIG. 9. Simultaneous traces of the velocity vs. time at $x_{1}=8.0 \mathrm{~cm}$ and $x_{2}=8.5 \mathrm{~cm}$ behind the comb.

FIG. 10. The cross correlation $C_{12}(\tau, \Delta x)$ vs. delay time $\tau$ for several different values of $\Delta x$ (as labeled).

FIG. 11. The delay time $\tau_{M A X}$ for the maximum of the cross correlations in Fig. 10 vs. the transit time $\Delta x / U_{0}$. The solid line corresponds to $\tau_{M A X}=\Delta x / U_{0}$.

FIG. 12. The maximum value of the cross correlations in Fig. 10, $C_{12}^{M A X}$ vs. the downstream separation $\Delta x$. The arrow shows the evolution length $\delta_{e} \simeq 7 \mathrm{~cm}$ (see text).

FIG. 13. Experimental check of Taylor's "statistical hypothesis": a) second order structure function $S_{2}(r)$ taken at $x=8 \mathrm{~cm}$ using Taylor's hypothesis; b) fourth order structure function $S_{4}(r)$; c) sixth order structure function $S_{6}(r)$. The open squares are direct spatial measurements made using two probes, and the lines correspond to the fitted scalings described in the text.

FIG. 14. Overlay of the velocity measured at $x_{1}=8.0 \mathrm{~cm}$ vs. $t$, and the velocity measured at $x_{1}=10.0 \mathrm{~cm}$ vs. $t-\tau_{M A X}$. The arrows indicate fluctuation spikes which have either appeared or disappeared during the transit between the two probes.

FIG. 15. A comparison of the coherence spectrum (Eq. 3) for $\Delta x=0.2 \mathrm{~cm}$, and the power spectrum taken at $x_{1}=8 \mathrm{~cm}$. The line is a fit to the power law $P s_{1}(f) \sim f^{-\alpha}$ with $\alpha=3.6 \pm 0.2$.

FIG. 16. Coherence spectra for several values of $\Delta x$ as a linear-log plot of $f$.

FIG. 17. Coherence spectra as in Fig. 16 normalized by the maximum cross correlation $C_{12}^{M A X}$ from Fig. 12. The straight lines through the high frequency part of the coherence spectra corresponds to a logarithmic decay law $C s(f) \sim \log (1 / f)$. 
FIG. 18. Coherence spectra for several large values of $\Delta x$ as a linear-log plot of $f$, normalized by the maximum cross correlation $C_{12}^{M A X}$ from Fig. 12 .

FIG. 19. The frequency scale $f_{d}$ characterizing the logarithmic decay $C s(f) \sim \log (1 / f)$ (lines in Fig. 17) vs. the separation $\Delta x$. The straight line represents the scaling law $f_{d} \sim \Delta x^{-1 / 2}$.

FIG. 20. The normalized error energy $\rho\left(\Delta x / U_{0}\right)$ as a function of time $\Delta x / U_{0}$ past the reference point $x_{1}$, shown as a log-linear plot. The arrow indicates the evolution time $T_{e}$ as defined in the text. Inset: an expanded view near the origin.

FIG. 21. A log-log plot of $\rho\left(\Delta x / U_{0}\right)$ as a function of downstream time $\Delta x / U_{0}$. The straight line corresponds to a power law dependence $\left(\Delta x / U_{0}\right)^{1 / 2}$ (see the text).

FIG. 22. A replot of $C_{12}^{M A X}$ from Fig. 12 as a function of probe separation $\Delta x$ normalized by the integral scale $\ell_{0}$. Also shown for comparison are $3 \mathrm{D}$ results from Ref. [21], Ref. [22], and Ref. 23.

FIG. 23. Gaussian fit to the shape of $C_{12}(\tau)$ at $\Delta x=4 \mathrm{~cm}$.

FIG. 24. An overlay of several $C_{12}(\tau)$ at various $\Delta x$ (as shown), plotted vs. $\tau-\tau_{M A X}$. 


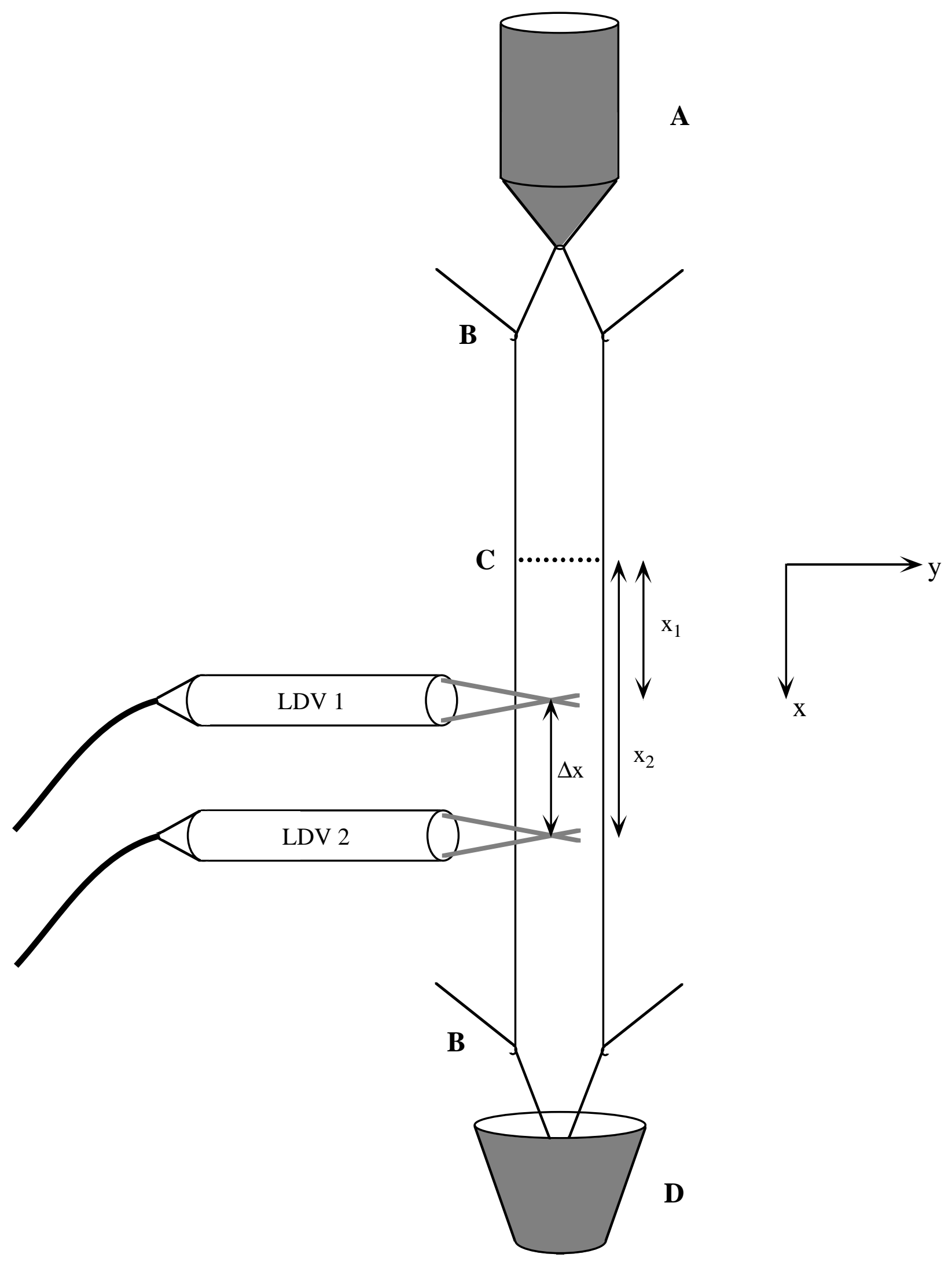



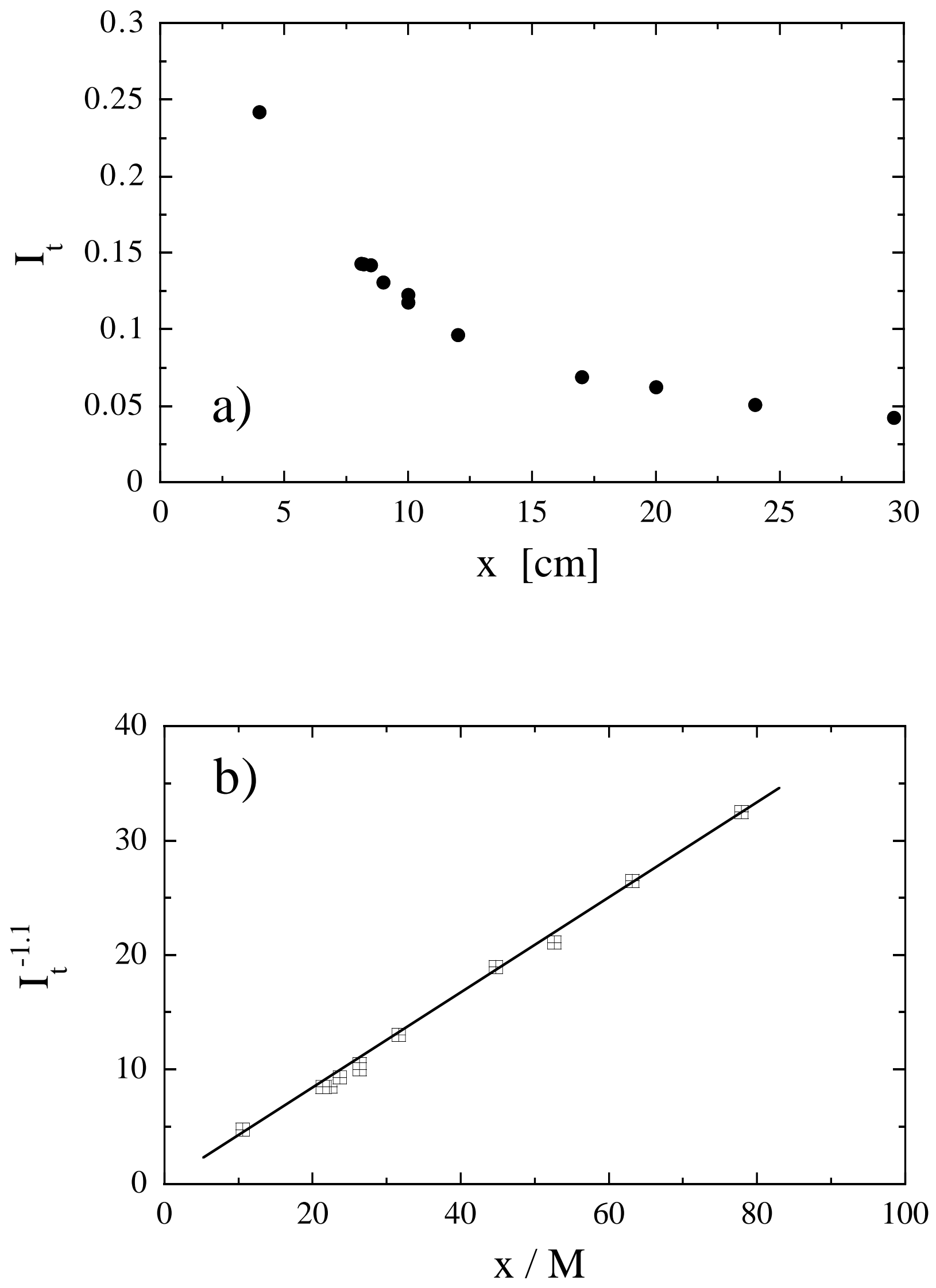


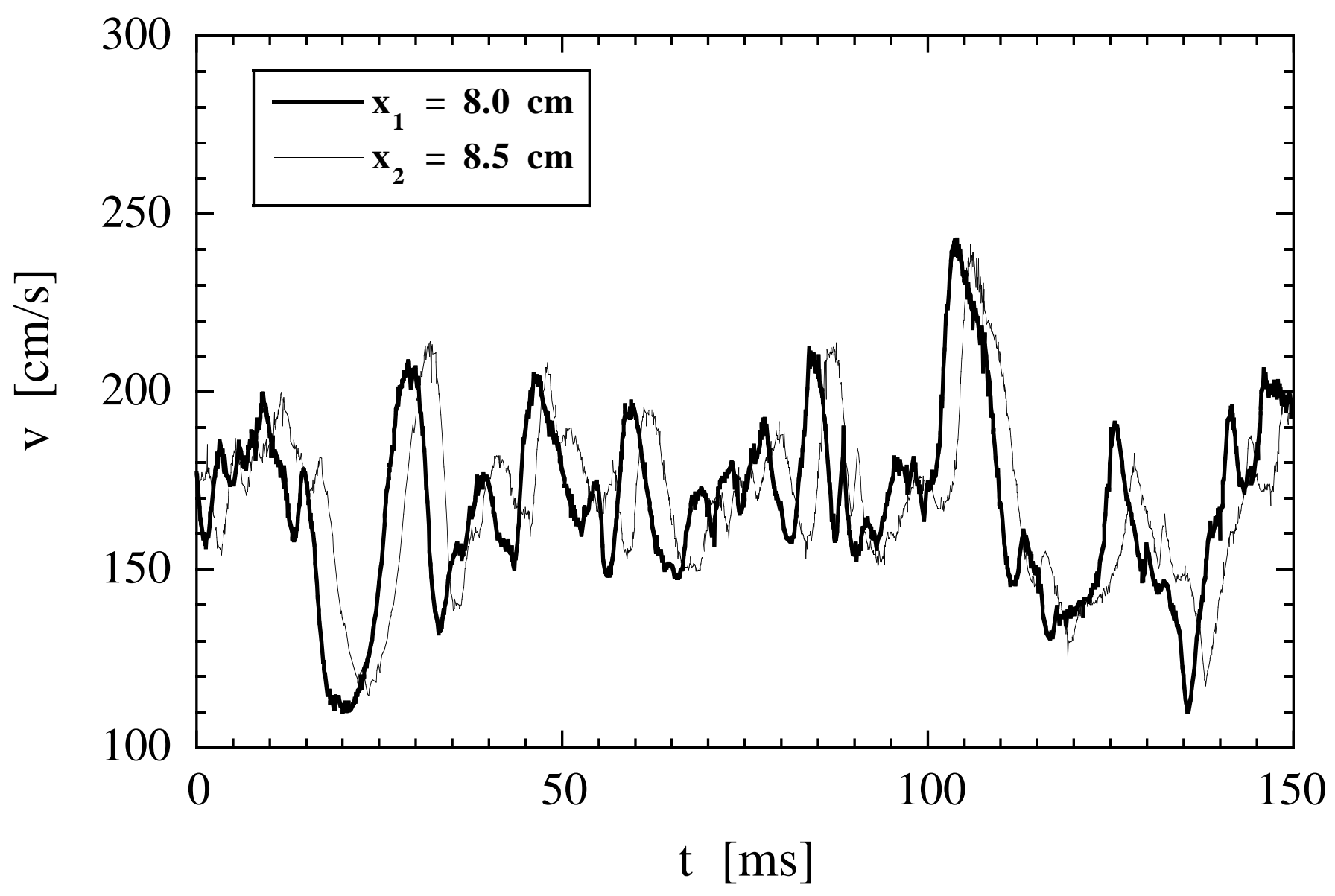




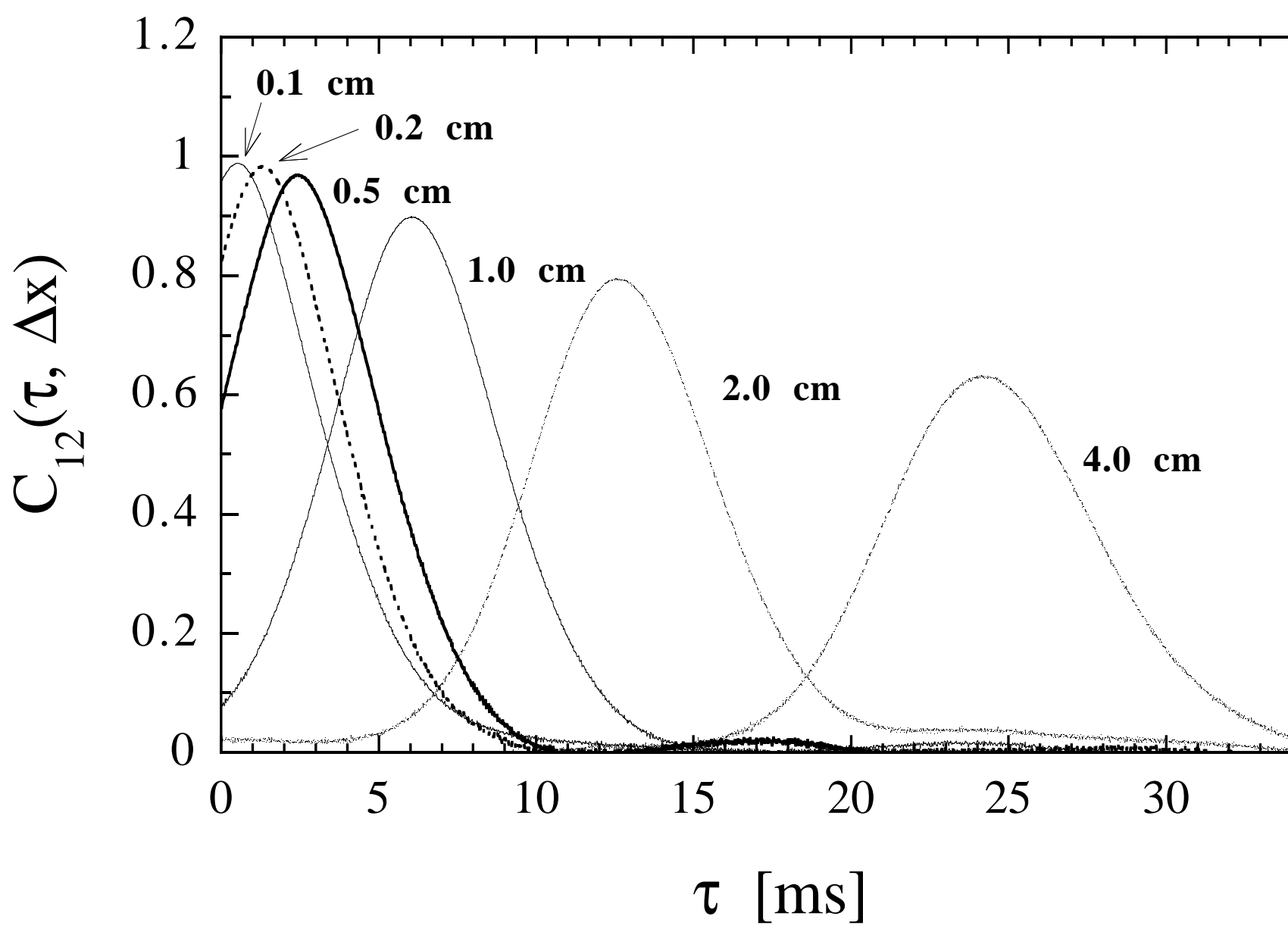




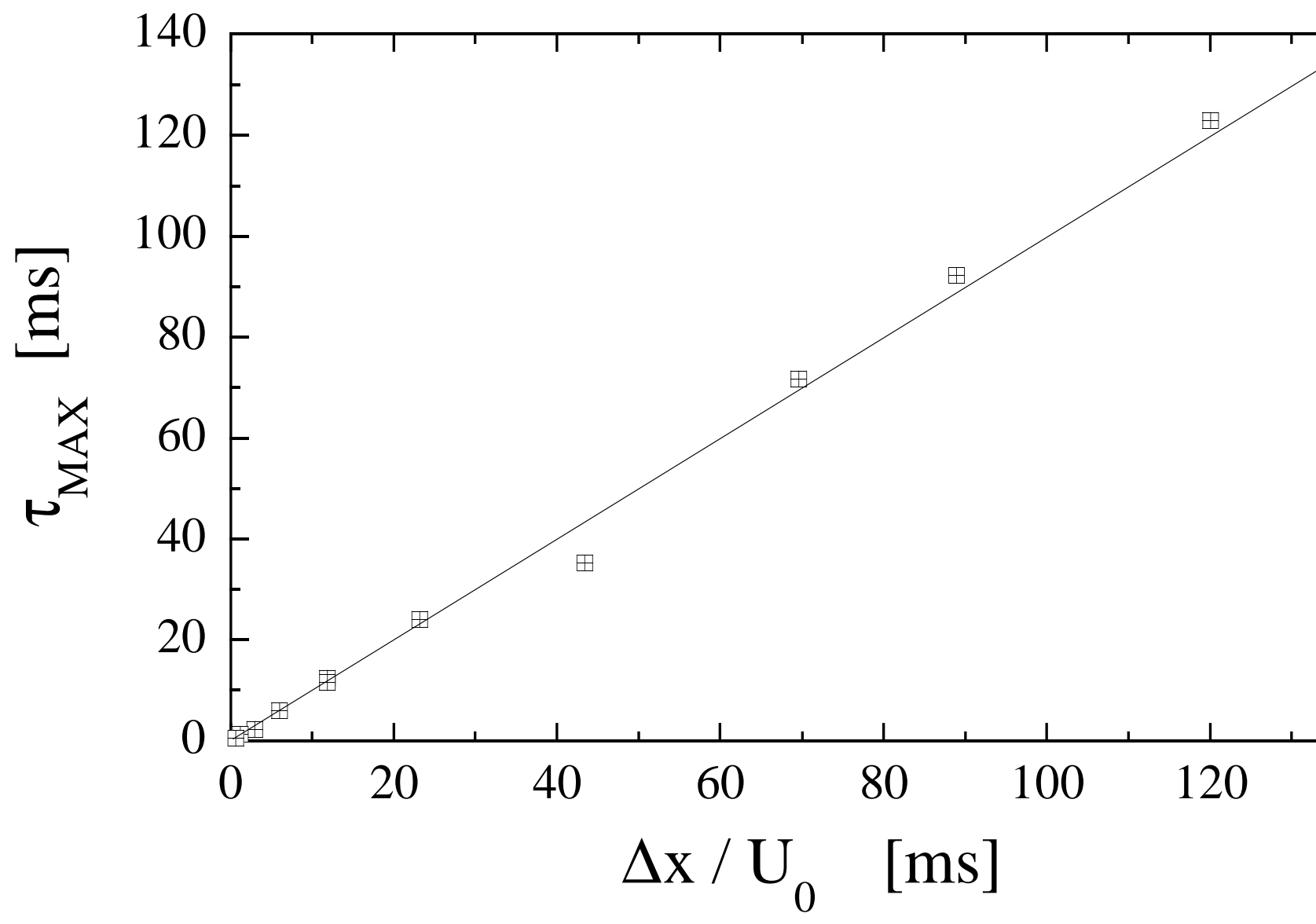




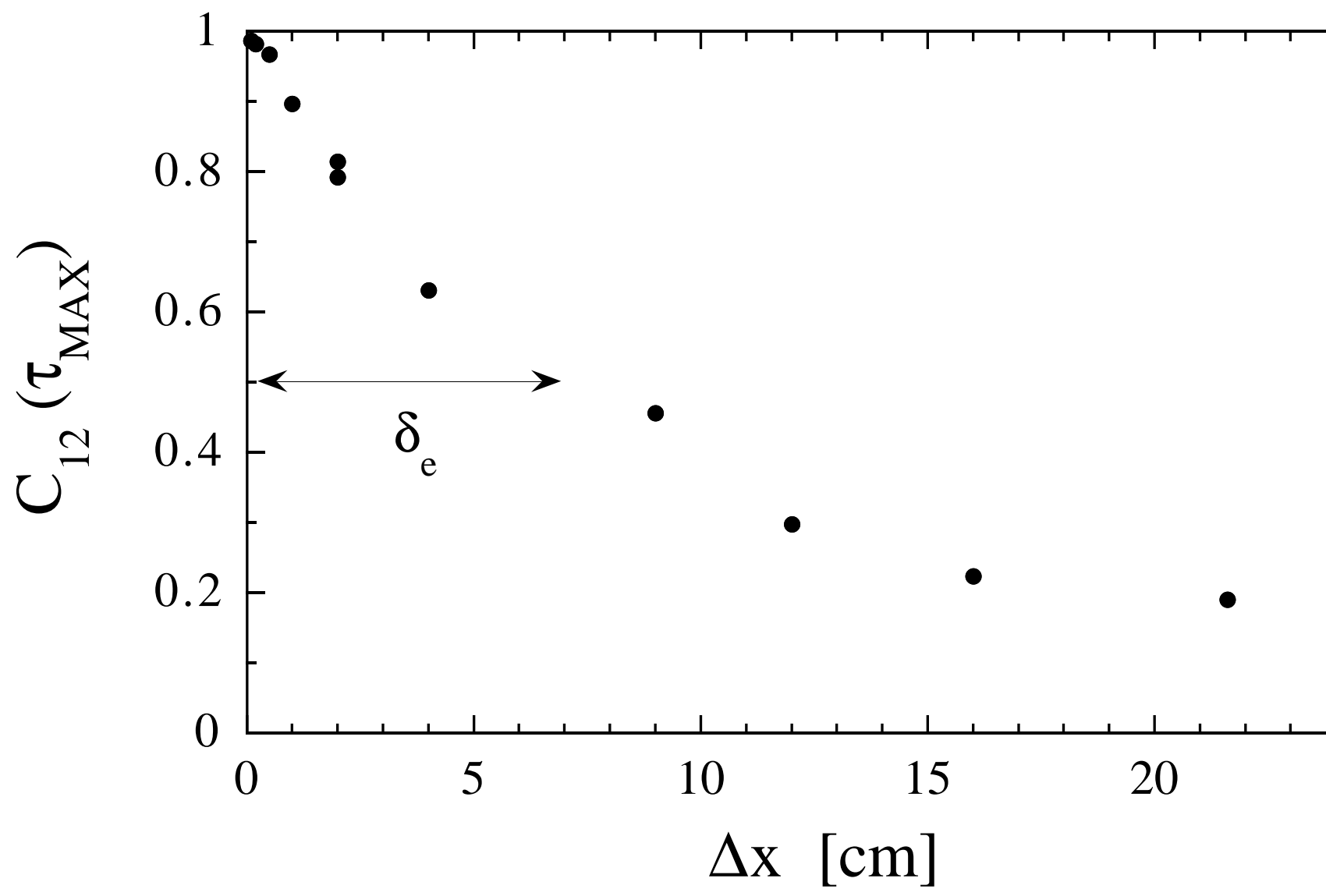



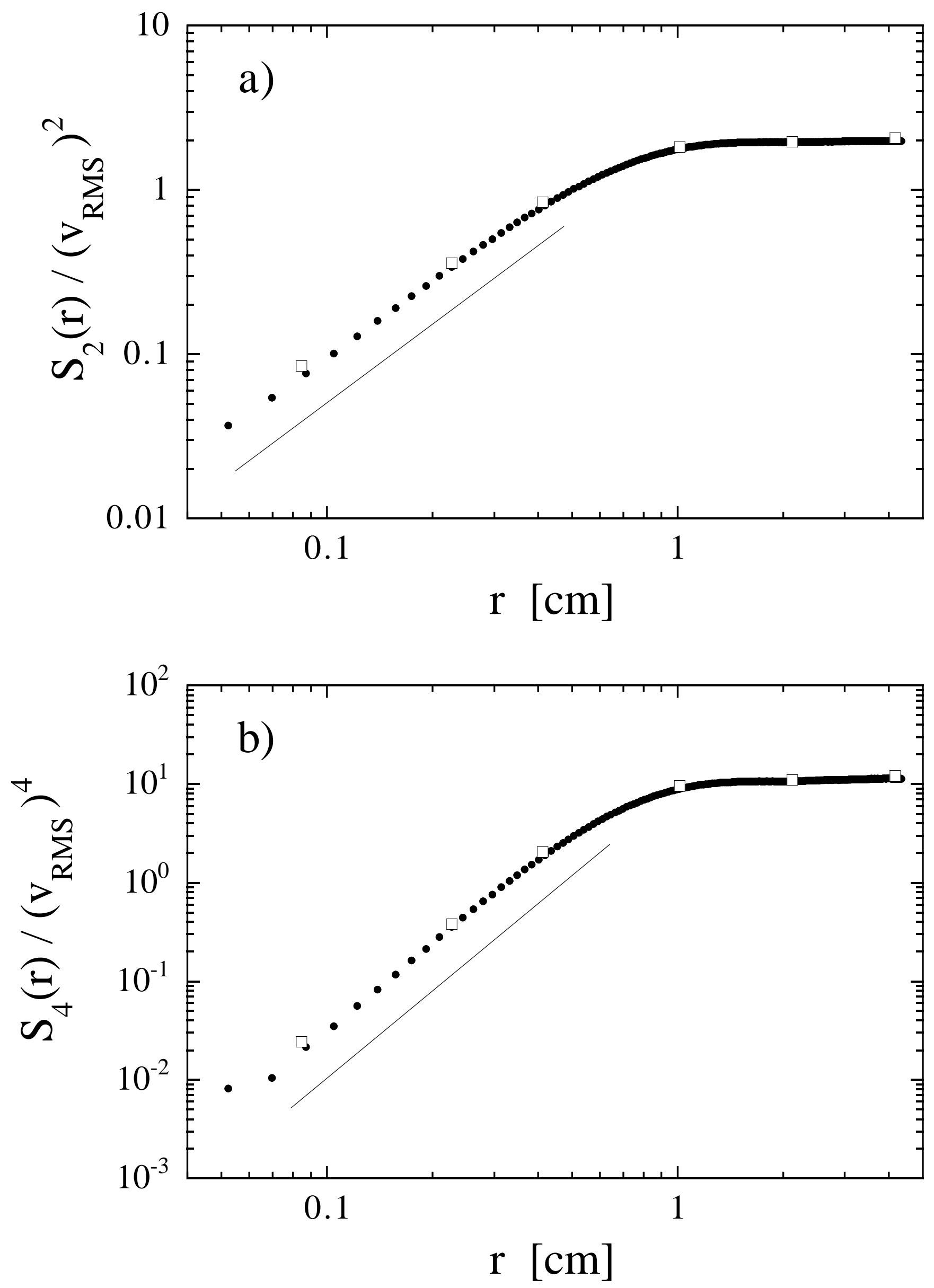


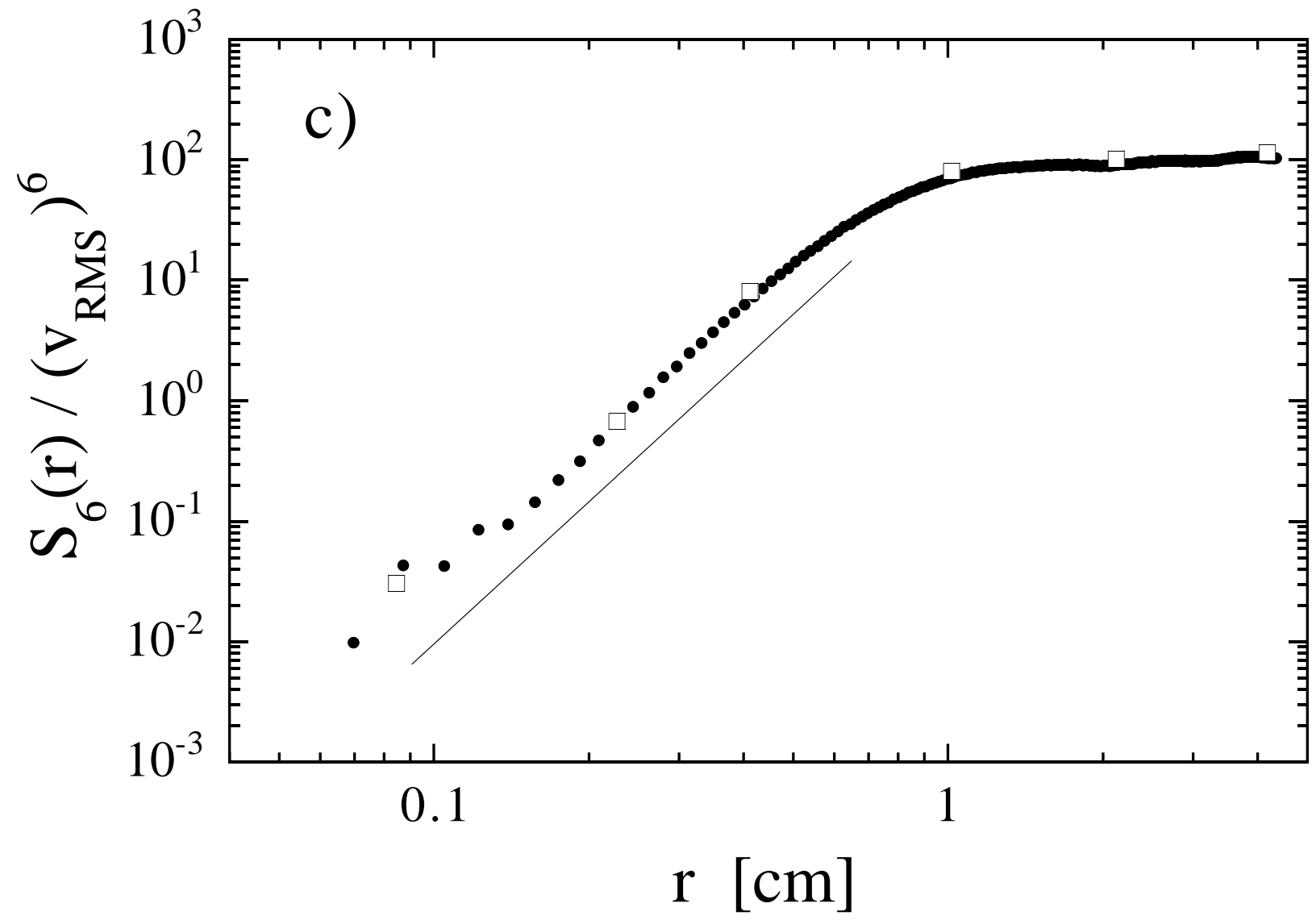




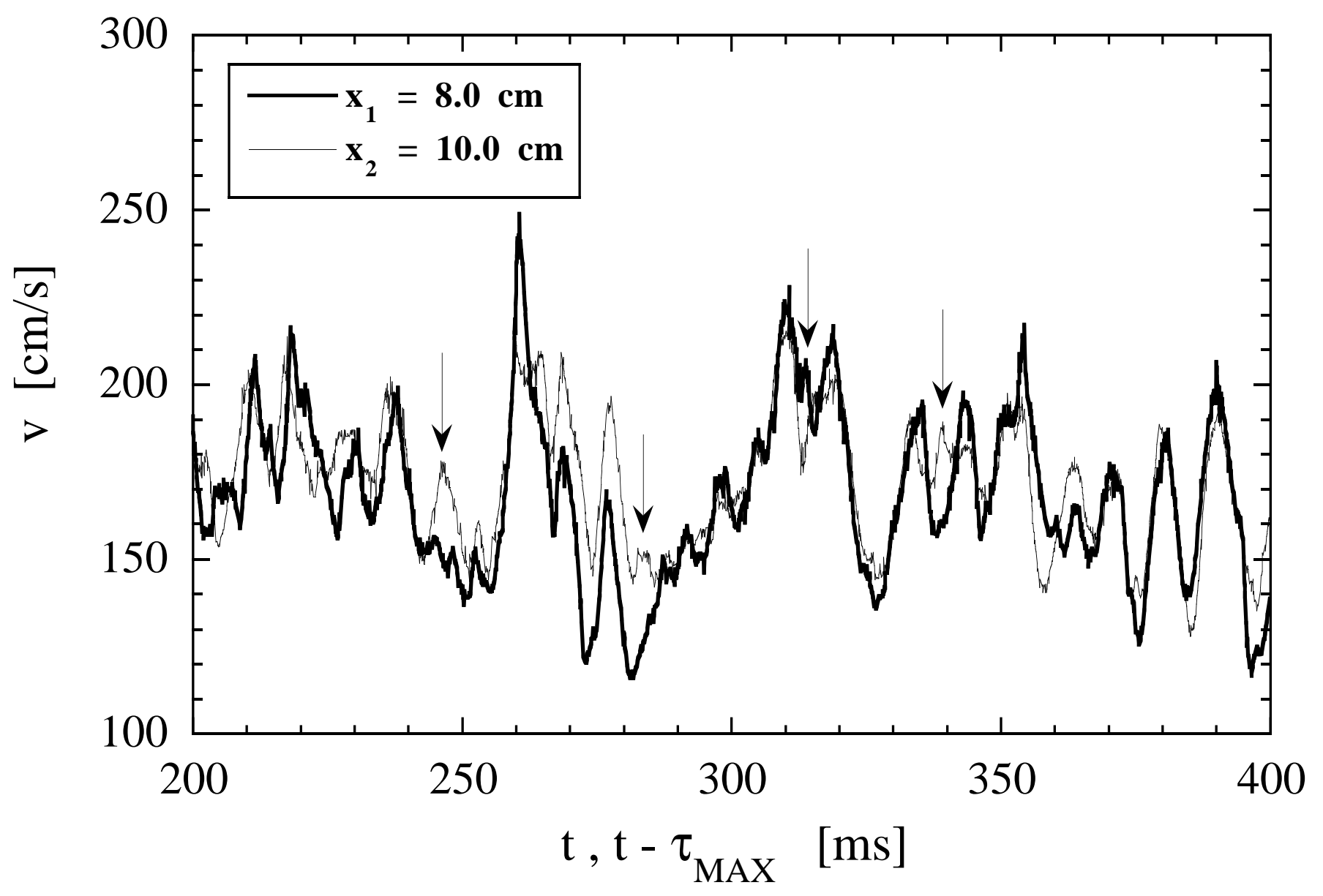




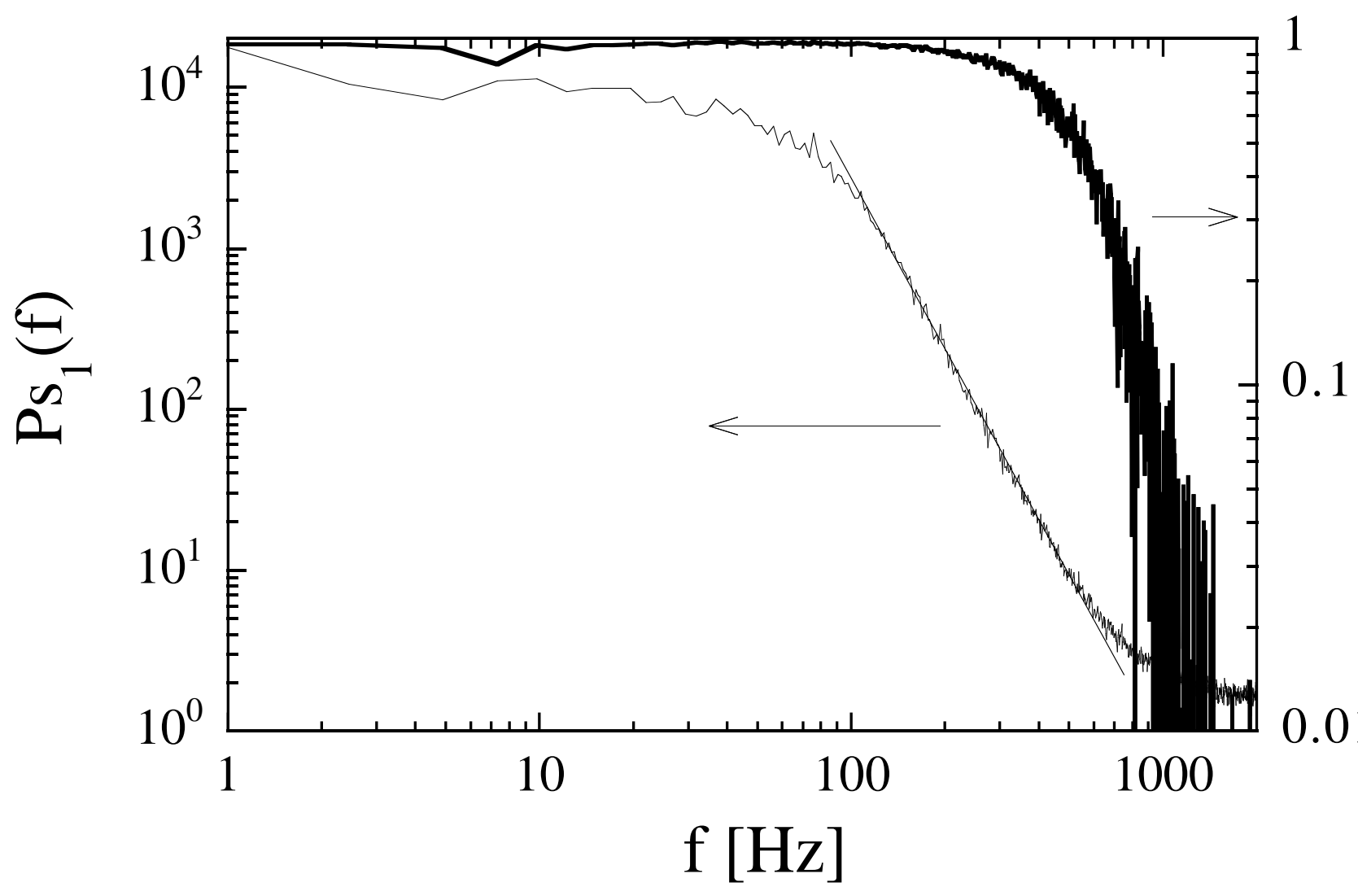




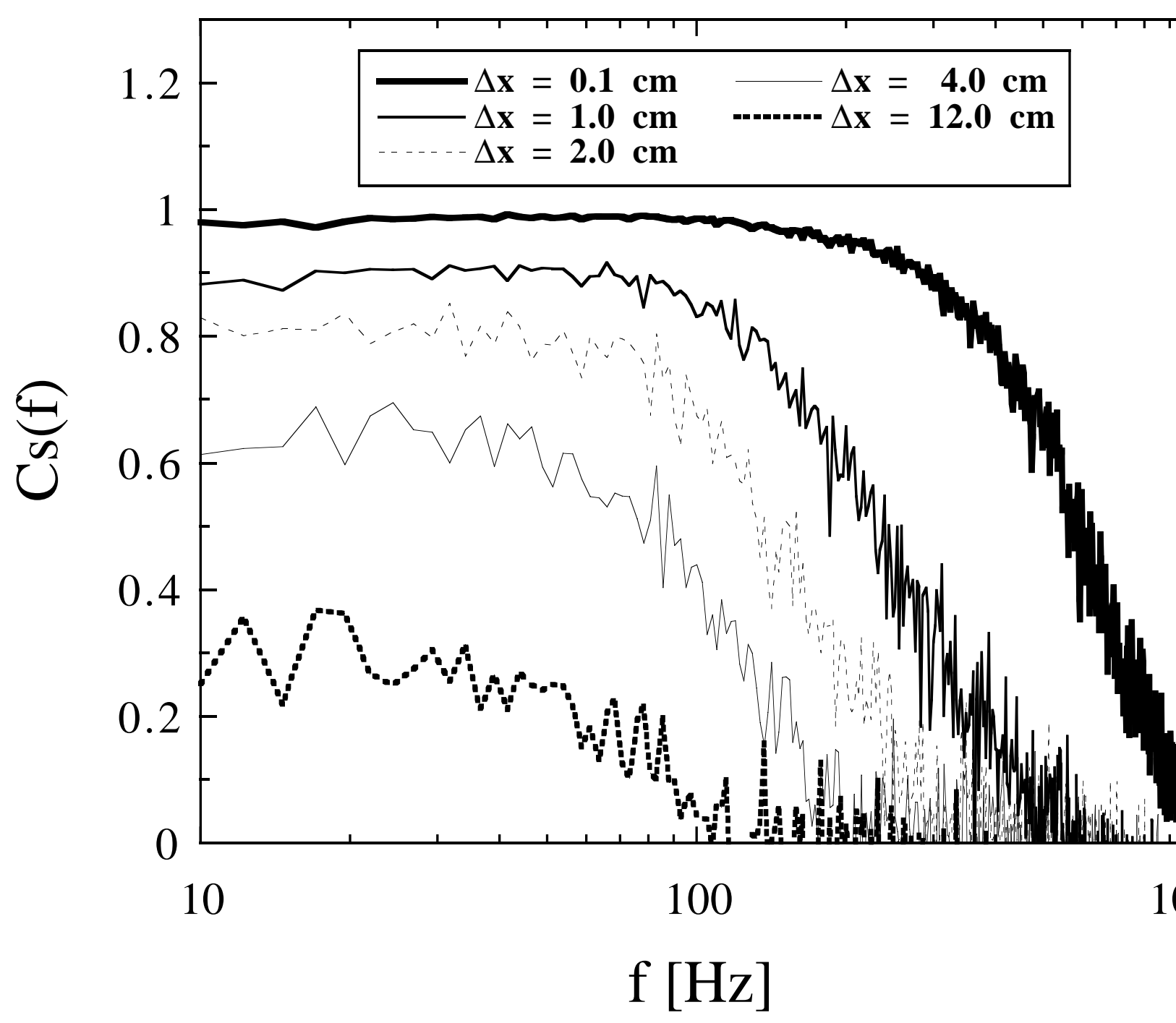




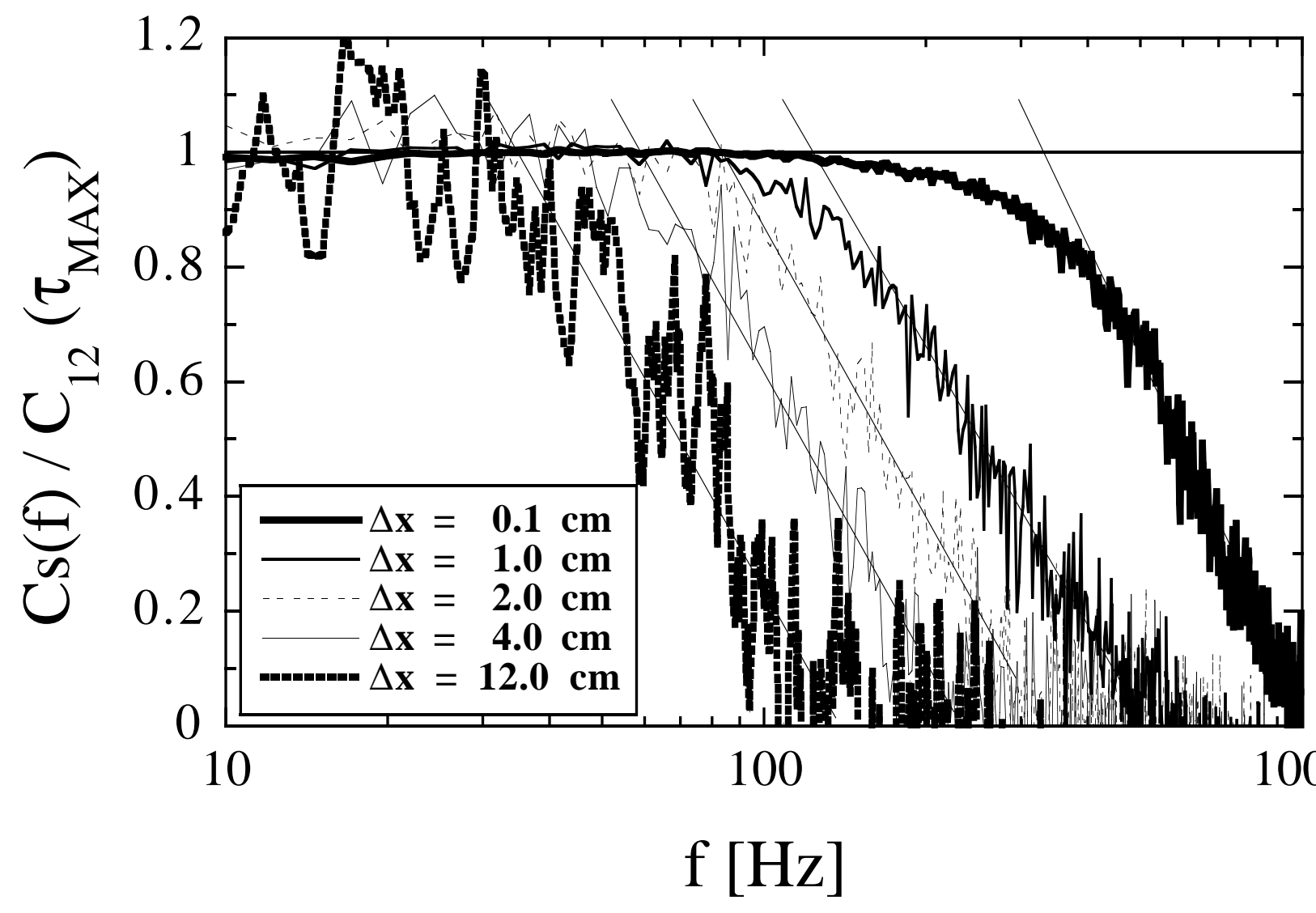




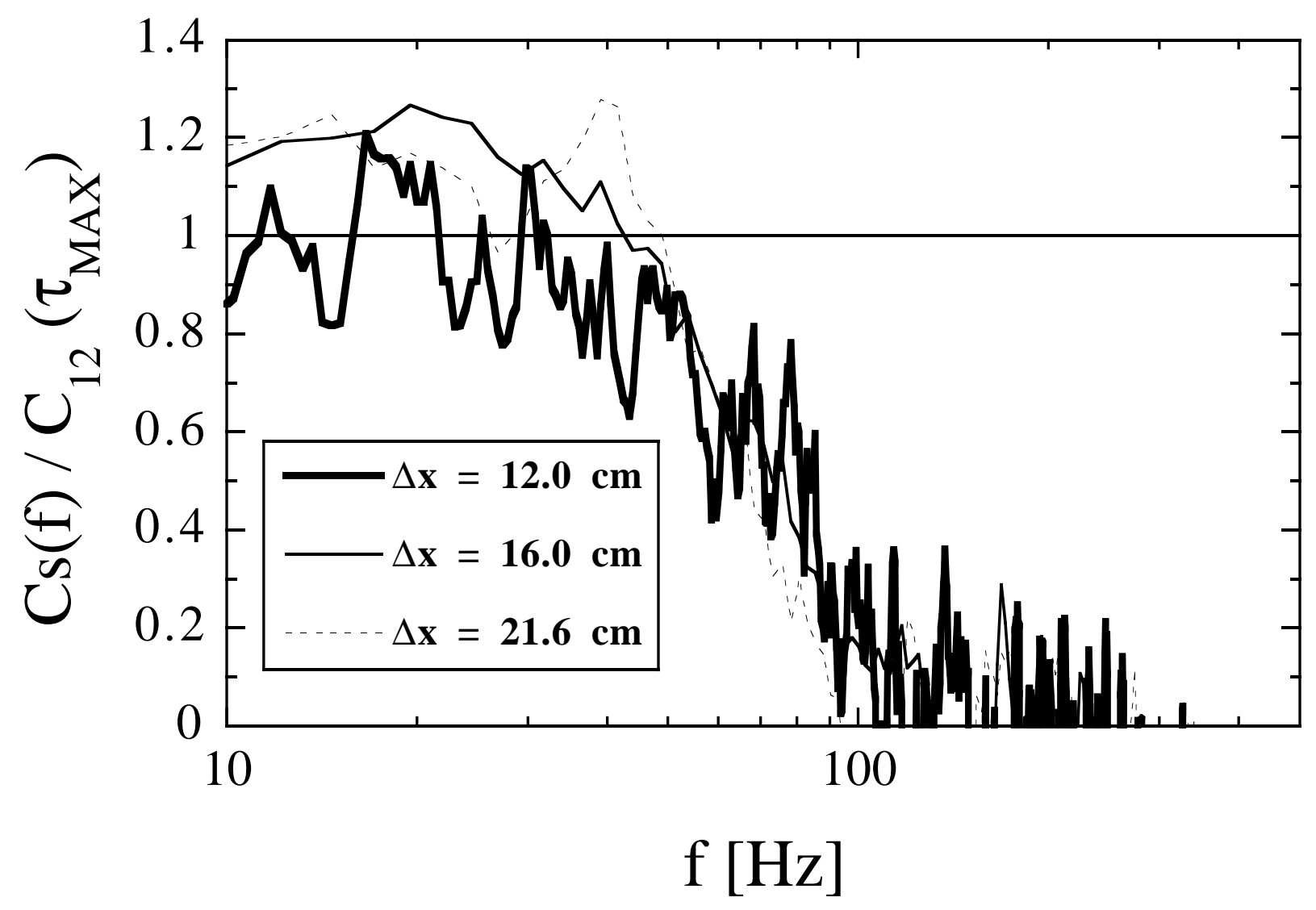




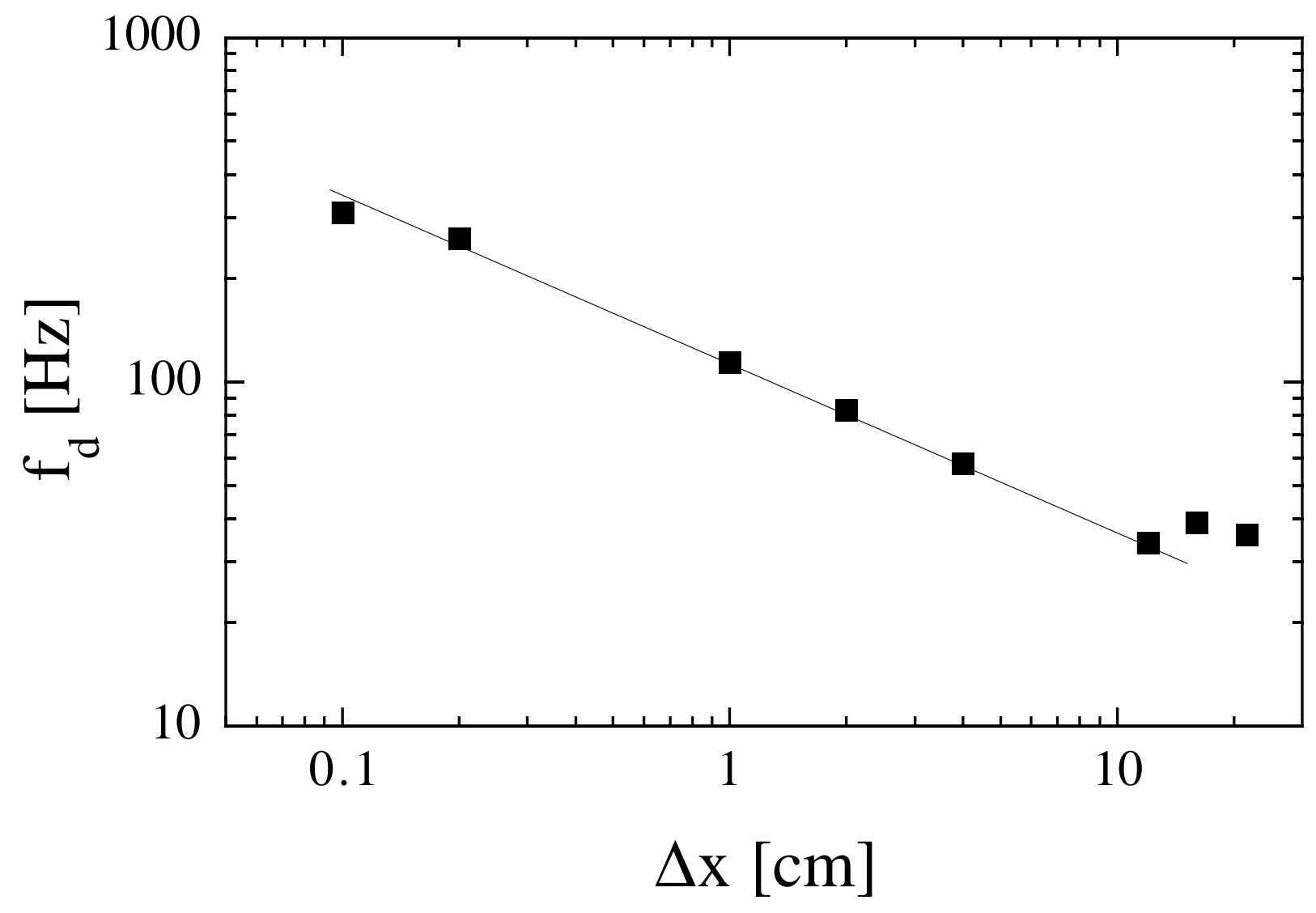




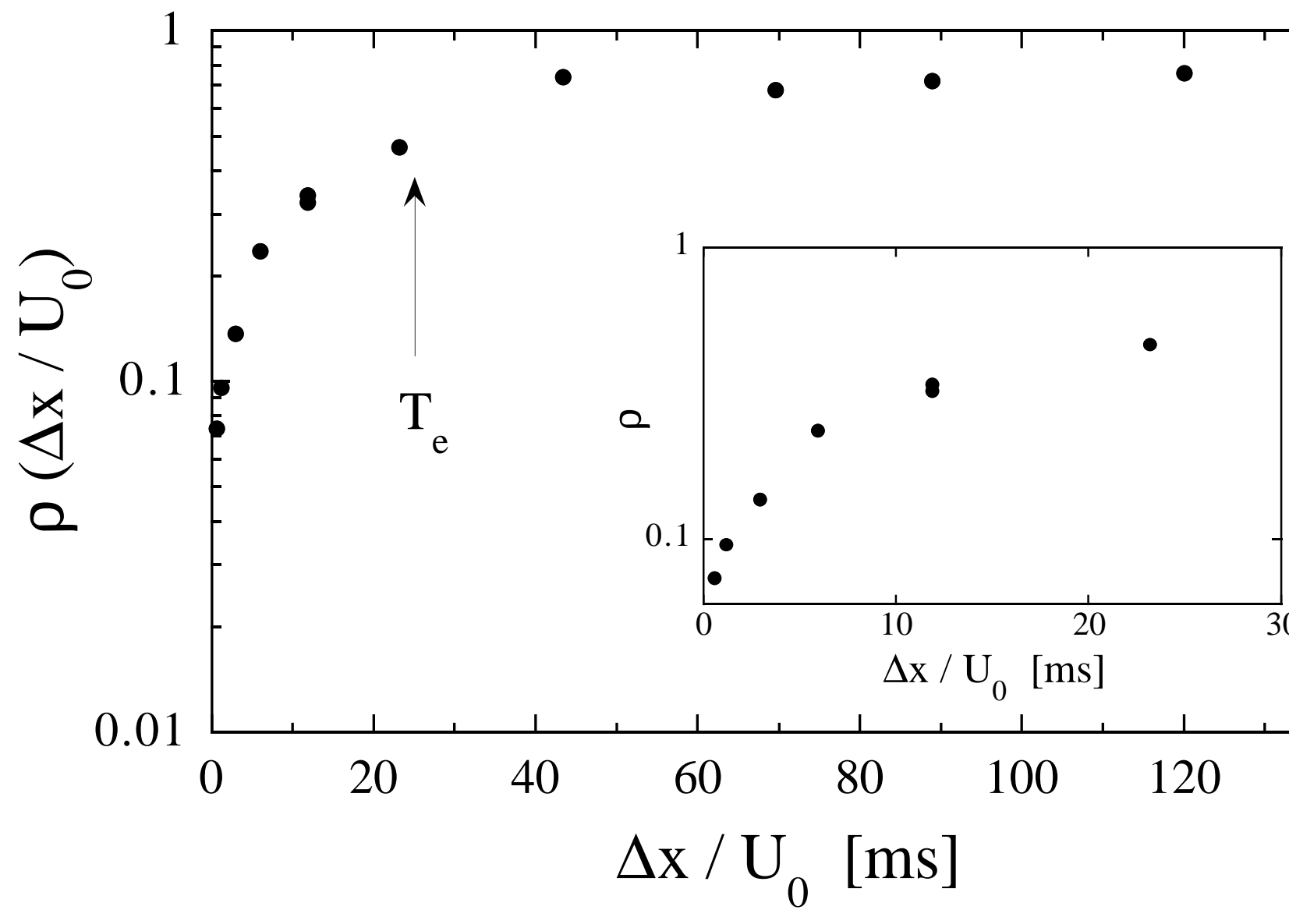




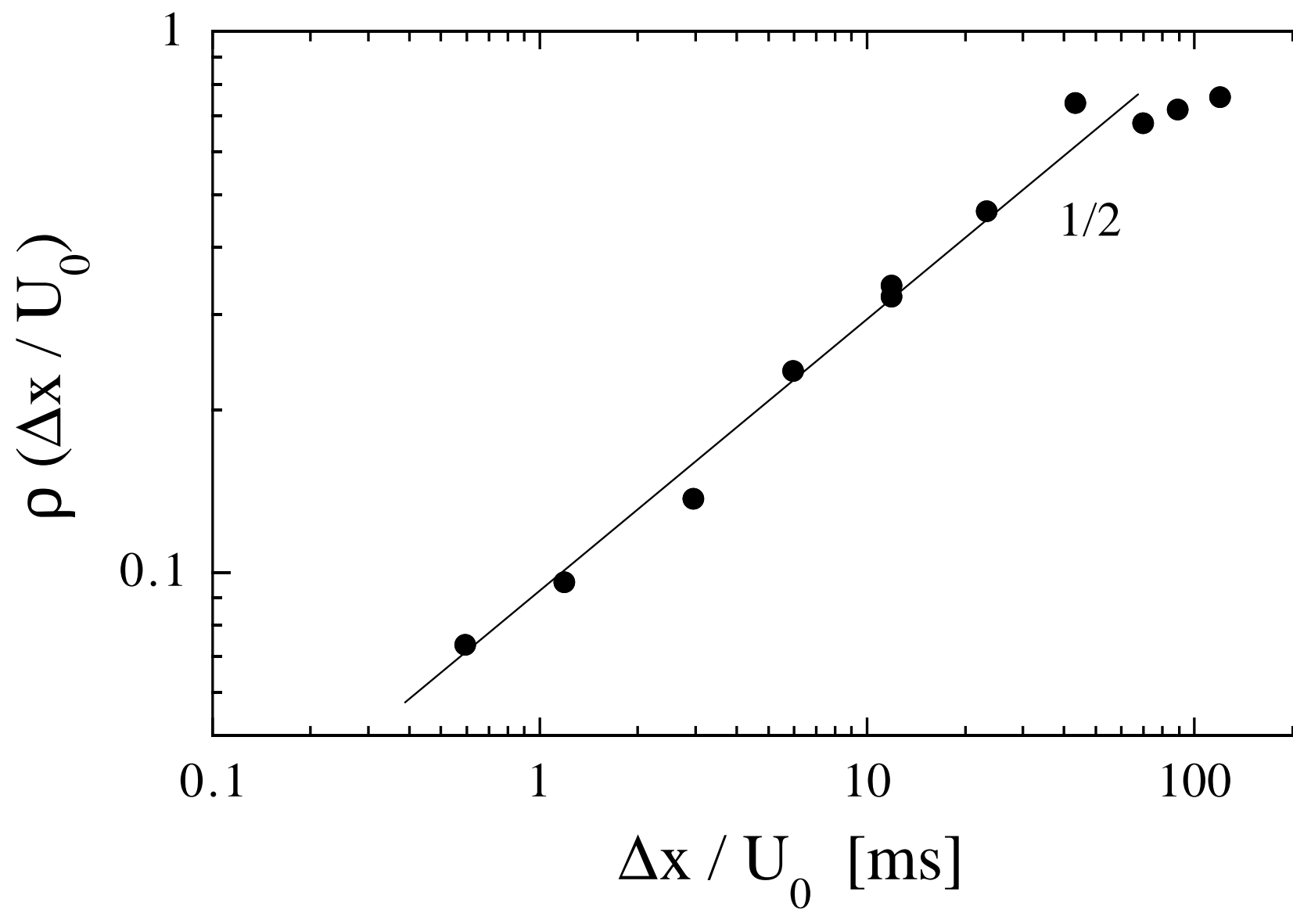




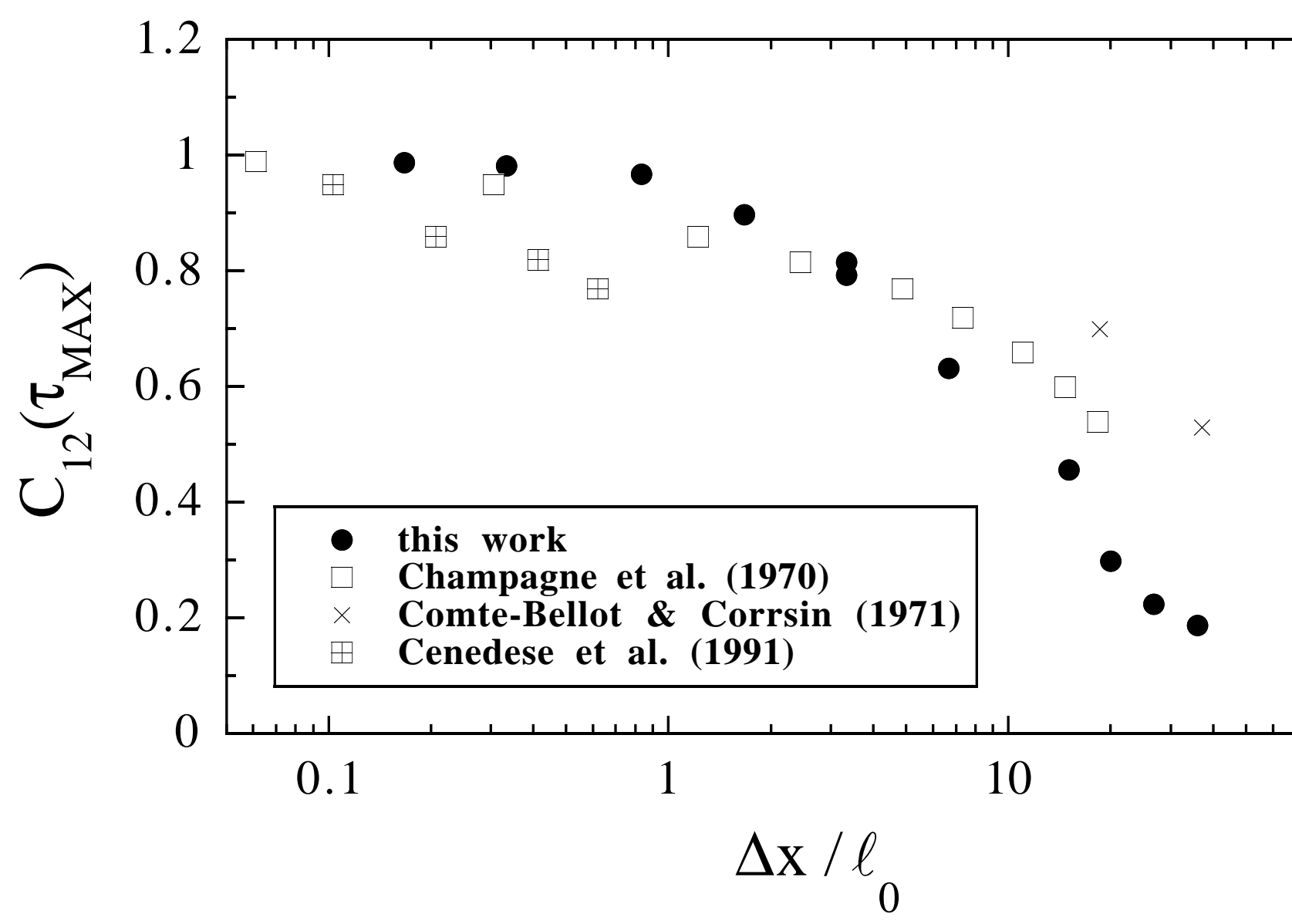




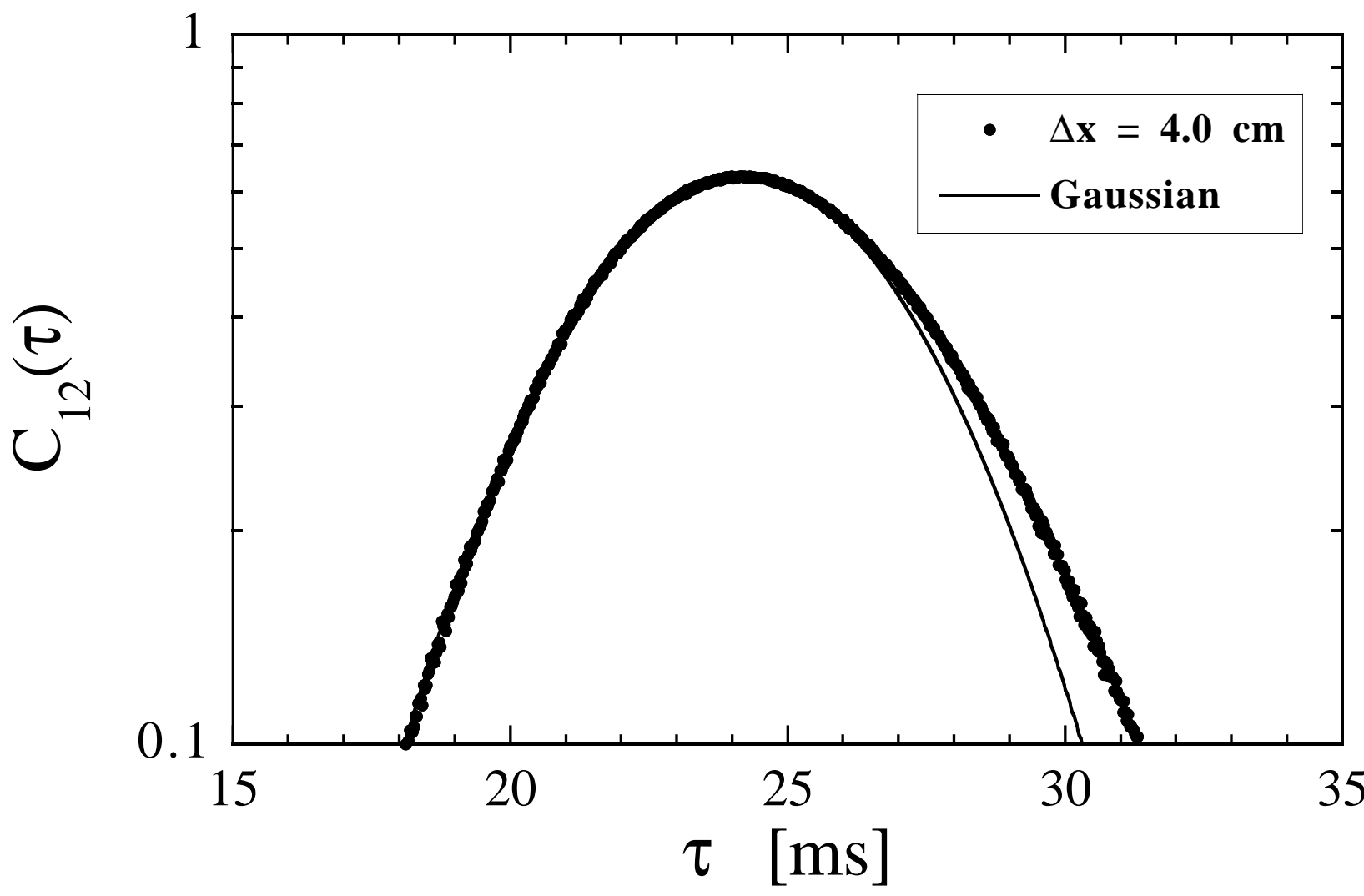




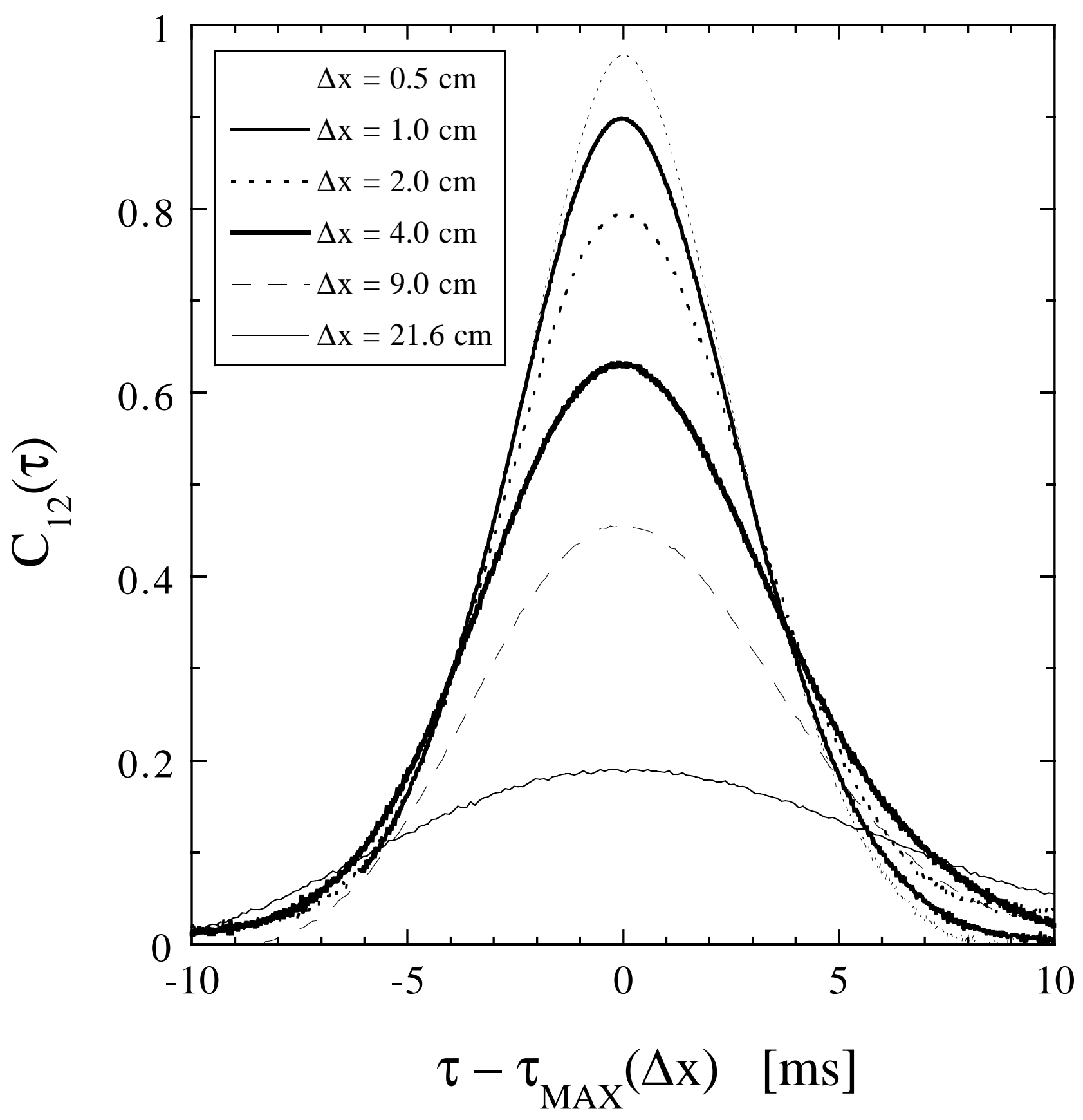

